BNL-113341-2016-JA

File \# 94294

\title{
Surface Profile and Stress Field Evaluation using Digital Gradient Sensing Method
}

\author{
C. Miao, B.M. Sundaram, L. Huang and H.V. Tippur
}

Submitted to Measurement Science \& Technology

Sept 1, 2016

Photon Sciences Department

Brookhaven National Laboratory

\author{
U.S. Department of Energy \\ USDOE Office of Science (SC), \\ Basic Energy Sciences (BES) (SC-22)
}

\footnotetext{
Notice: This manuscript has been authored by employees of Brookhaven Science Associates, LLC under Contract No. DE- SC0012704 with the U.S. Department of Energy. The publisher by accepting the manuscript for publication acknowledges that the United States Government retains a non-exclusive, paid-up, irrevocable, world-wide license to publish or reproduce the published form of this manuscript, or allow others to do so, for United States Government purposes.
} 


\section{DISCLAIMER}

This report was prepared as an account of work sponsored by an agency of the United States Government. Neither the United States Government nor any agency thereof, nor any of their employees, nor any of their contractors, subcontractors, or their employees, makes any warranty, express or implied, or assumes any legal liability or responsibility for the accuracy, completeness, or any third party's use or the results of such use of any information, apparatus, product, or process disclosed, or represents that its use would not infringe privately owned rights. Reference herein to any specific commercial product, process, or service by trade name, trademark, manufacturer, or otherwise, does not necessarily constitute or imply its endorsement, recommendation, or favoring by the United States Government or any agency thereof or its contractors or subcontractors. The views and opinions of authors expressed herein do not necessarily state or reflect those of the United States Government or any agency thereof. 


\title{
Surface Profile and Stress Field Evaluation using Digital Gradient Sensing Method
}

\author{
C. Miao ${ }^{1}$, B.M. Sundaram ${ }^{1}$, L. Huang ${ }^{2}$ and H.V. Tippur ${ }^{1 *}$ \\ ${ }^{1}$ Department of Mechanical Engineering, Auburn University, AL 36849, USA \\ ${ }^{2}$ Brookhaven National Laboratories, Upton, NY 11973, USA
}

\begin{abstract}
Shape and surface topography evaluation from measured orthogonal slope/gradient data is of considerable engineering significance since many full-field optical sensors and interferometers readily output such a data accurately. This has applications ranging from metrology of optical and electronic elements (lenses, silicon wafers, thin film coatings), surface profile estimation, wave front and shape reconstruction, to name a few. In this context, a new methodology for surface profile and stress field determination based on a recently introduced non-contact, fullfield optical method called Digital Gradient Sensing (DGS) capable of measuring small angular deflections of light rays coupled with a robust Finite-difference-based Least-squares Integration (HFLI) scheme in the Southwell configuration is advanced here. The method is demonstrated by evaluating (a) surface profiles of mechanically warped silicon wafers and (b) stress gradients near growing cracks in planar phase objects.
\end{abstract}

Keywords: Optical Metrology; Surface Slopes; Stress Gradients; Digital Gradient Sensing; Surface Topography; Stress Evaluation

\section{Introduction}

There is a demand for an accurate, non-contact evaluation of surface topography of objects. Some optical sensors such as the Shack-Hartmann wave-front sensor [1], the lateral shearing interferometers [2], and the pyramid sensor [3] have all been used for this purpose. The current literature indicates that it is more efficient for these sensors to just measure the surface gradients instead of measuring the values of absolute height due to channel capacity limitations [4]. Integration algorithms are applied then to convert the measured gradients into topographic information. It can be concluded that a highly accurate, non-contact method for measuring

\footnotetext{
* McWane Endowed Chair Professor and Corresponding Author, Tel: +1-334-844-3327, email: tippuhv@auburn.edu
} 
surface gradients in combination with an accurate integration method are among the key factors to satisfactorily reconstruct surface topography.

As already noted, several optical metrology techniques for measuring surface gradients currently exist. Based on the type of illumination used, they can be divided into two categories, coherent (interferometric) and incoherent (moiré) methods [5, 6, 7, 8, 9]. In these, measurements are typically in the form of optical fringes and are analog in nature. Further, orthogonal gradients are generally evaluated by performing multiple or successive experiments (e.g., Coherent Gradient Sensing or CGS method [7, 8]) which may require altering the setup. Also, the optical phase evaluation using phase shifting methods is often needed to determine gradients in the digital form. In recent years, however, digital image correlation (DIC) methods have become rather popular for measuring deformations, two or all three orthogonal displacements in the whole field, as they offer many advantages besides directly providing the required information in the digital form [10, 11, 12, 13]. By taking advantage of this, a new full-field optical method called Digital Gradient Sensing (DGS) method has been proposed recently for measuring two orthogonal small angular deflections of light rays caused by stresses in planar solids [14, 15]. Subsequently, the method has also been extended to study optically reflective objects as well [16]. The simplicity of experimental setup, its accuracy and robustness make DGS attractive for measuring two orthogonal surface slopes and stress gradients in the whole field. Subsequent quantification of surface profile or stresses from these measurements via two-dimensional integration of measured data is valuable in many engineering applications.

There are several two-dimensional integration methods reported in the literature. Broadly, they can be divided into two types: path dependent integration methods $[17,18,19]$ and path independent integration methods [4, 20, 21, 22, 23, 24]. Path dependent integration methods such as trapezoidal rule or Simpson's rule calculate the local height increments by directly integrating the function. These methods are easy to implement due to the simplicity and efficiency in computing. However, the accuracy of results from these methods depends heavily on integration paths. Noise in measured data can propagate and accumulate along the integration path. Such errors can be mitigated in path independent integration methods because gradient data have global influence on the solution procedures and errors get uniformly dispersed over the whole surface [25]. 
Methods based on Fourier transforms [20] are a family of path independent integration methods which are fast and accurate. However, these methods cannot deal with irregular surface shapes which make it not very practical. Another family of path independent integration methods, which can also be fast and highly accurate, is least-squares based integration methods [21, 22, 23 , 24]. Hudgin [21] proposed a least-squares recursive algorithm for wave-front reconstruction from phase difference and studied the propagation of noise. Southwell [22] proposed a more detailed least-squares integration algorithm for wave-front reconstruction based on a rectangular grid configuration. Huang et al. [23] pointed out the assumption of biquadratic functions in Southwell grid configuration limited the algorithm. They proposed an optimized algorithm by implementing iterative compensations. Li et al. [24] pointed out that Huang's algorithm was rather time consuming although the accuracy of reconstruction was significantly improved, and hence proposed a more accurate and less time intense approach. Ettl et al. [4] proposed another kind of integration method by using radial basis functions which showed robustness and high accuracy. However, compared with Li's method, it was found time consuming, especially when the dataset is incomplete. Huang et al. [26] compared three families of two-dimensional integration methods: (a) the Finite-difference-based least-squares integration methods, (b) the transform-based integration methods and (c) the Radial basis function based integration methods. Their performance, advantages and weaknesses are discussed in details for specific cases. Accordingly, Li et al.'s algorithm [24] is selected in the current work.

In the following, the experimental details and working principle of both reflection-mode DGS (r-DGS) and transmission-mode DGS (t-DGS) are briefly presented. Next, the algorithm of the two-dimensional integration method is explained along with its verification using an idealized function. Then, the feasibility of the two-dimensional integration method in conjunction with r-DGS is discussed in detail using a Si wafer subjected to central and eccentric out-of-plane loading. This is followed by the demonstration of extracting stress fields from measured stress gradients from t-DGS. Finally, the major results of this study are summarized.

\section{Experimental setup and working principle}

\subsection{Reflection-mode Digital Gradient Sensing (r-DGS)}

A schematic of the experimental setup for reflection-mode Digital Gradient Sensing (r-DGS) used to measure surface slopes is shown in Fig. 1. A digital camera, a reflective planar specimen, 
a micrometer, a beam splitter and a target plate are included in the figure. The target plate is decorated with random speckles using alternate mists of black and white paints. The beam splitter is placed between the specimen and target plate at an angle of $45^{\circ}$ to the optical axis of the setup for the camera to photograph the speckles on the target via the specimen surface. The target plate is illuminated by a pair of cool LED lamps emitting white light.

For simplicity, the angular deflections of light rays only in the $y$-z plane are shown in Fig. 2. Initially, a point $\mathrm{P}$ on the target plate is recorded by the camera through a point $\mathrm{O}$ when the specimen is in its undeformed or reference state. When the specimen suffers out-of-plane deformation, a neighboring point $\mathrm{Q}$ on the target gets recorded by the camera through $\mathrm{O}$. OP makes an angle $\phi_{y}$ with OQ and $\phi_{y}=\theta_{i}+\theta_{r}$ where $\theta_{i}$ and $\theta_{r}\left(=\theta_{i}\right)$ are incident and reflected angles relative to the normal to the specimen. The two orthogonal surface slopes can be expressed as $\frac{\partial w}{\partial y: x}=\frac{1}{2} \tan \left(\phi_{y: x}\right)$. The local displacements $\delta_{y: x}$ can be measured by correlating the undeformed image of the specimen with respect to the deformed image. Finally, the two orthogonal surface slopes can be determined using [16]:

$$
\frac{\partial w}{\partial y: x}=\frac{1}{2} \tan \left(\phi_{y: x}\right) \approx \frac{1}{2}\left(\phi_{y: x}\right) \approx \frac{1}{2}\left(\frac{\delta_{y: x}}{\Delta}\right)
$$

where $\Delta$ is the distance between the specimen and target plate and small angle approximation is evoked.

The experimental parameters including $\Delta$, speckle characteristics on target plate, subimage size used for image correlation control the accuracy of the method and the details can be found in Ref. [27]. Further, it should be noted here that the camera is focused on the target plane and the coordinates of the specimen plane are used in the analysis subsequently. Therefore, based on the pin-hole camera approximation, a mapping function is used to transfer the information to the specimen plane as, $(x: y)=\frac{L}{L+\Delta}\left(x_{0}: y_{0}\right)$ where $(x: y)$ and $\left(x_{0}: y_{0}\right)$ denote the coordinates of the specimen and target planes, respectively, and $L$ is the distance between the specimen and camera [14].

\subsection{Transmission-mode Digital Gradient Sensing (t-DGS)}


A schematic of the experimental setup for transmission-mode digital gradient sensing (t-DGS) method is shown in Fig. 3. Unlike r-DGS, in t-DGS a random speckle pattern on target plate is recorded through a transparent specimen. White light is used here to illuminate the target. As in r-DGS, an undeformed or reference image is recorded first. That is, point $\mathrm{P}$ on the target plate which corresponds to point $\mathrm{O}$ on the specimen plane is recorded. The refractive index and thickness of the specimen change after imposing a load on the specimen. As a result, light rays from the initial path, which is referred to as the elasto-optical effect. In the deformed state of the specimen, an image is recorded. That is, a neighboring point of $\mathrm{P}$, namely $\mathrm{Q}$ on the target plate is recorded through $\mathrm{O}$ on the specimen plane. The local displacements $\delta_{y: x}$ are measured by correlating the undeformed image with the deformed image of the specimen. The angular deflections of light rays $\phi_{y: x}$, which are related to in-plane stress gradients, can be expressed as [15]:

$\phi_{x: y} \approx \frac{\delta_{x: y}}{\Delta}=C_{\sigma} B \frac{\partial\left(\sigma_{x x}+\sigma_{y y}\right)}{\partial(x: y)}$

where $C_{\sigma}$ is the elasto-optical constant of the specimen material, $B$ is its initial thickness, $\Delta$ is the distance between the specimen and target plate, and $\sigma_{x x}+\sigma_{y y}$ is the sum of two in-plane normal stresses . Again, the pin-hole camera mapping function is used here to transfer coordinates of the target to the specimen.

\section{The 2D integration method}

The above two full-field optical methods, r-DGS and t-DGS, provide two orthogonal surface slopes and stress gradients in the region of interest as a rectangular array of data. Traditionally, surface topography and stresses in addition to or in place of surface slopes and stress gradients from such measurements are quite useful in engineering applications. Hence, reconstructing the same from DGS is valuable.

In the field of surface reconstruction from slope data, two-dimensional integration based on finite-difference is widely used to compute topographic values from gradient data by leastsquares procedures. Southwell proposed a Traditional Finite-difference-based Least-squares Integration (TFLI) algorithm for a grid configuration, as shown in Fig. 4, requiring the measured surface slopes and the evaluated topographic values are at the same spatial position [22, 26]. The 
relation between the slopes and the evaluated function values in a $M \times N$ matrix in this approach can be expressed as [26]:

$\begin{cases}\frac{f_{m, n+1}-f_{m, n}}{x_{m, n+1}-x_{m, n}}=\frac{s_{m, n+1}^{x}+s_{m, n}^{x},}{2} & m=1,2, \ldots, M, \\ \frac{f_{m+1, n}-f_{m, n}}{y_{m+1, n}-y_{m, n}}=\frac{s_{m+1, n}^{y}+s_{m, n}^{y}}{2}, & m=1,2, \ldots, N-1 .\end{cases}$

where $x, y$ are the local coordinates, $s$ denotes the local surface slopes, $f$ is the value of the function at $(x, y)$. Eq. (3) can be converted to a matrix form,

$$
D F=G
$$

where

$$
\boldsymbol{D}=\left[\begin{array}{c}
D^{x} \\
D^{y}
\end{array}\right]=\left[\begin{array}{ccccccccc}
-1 & 0 & \cdots & 0 & 1 & 0 & \cdots & \cdots & 0 \\
0 & -1 & 0 & \cdots & 0 & 1 & 0 & \cdots & 0 \\
\vdots & \vdots & \vdots & \vdots & \vdots & \vdots & \vdots & \vdots & \vdots \\
0 & \cdots & \cdots & 0 & -1 & 0 & \cdots & 0 & 1 \\
\hdashline-1 & 1 & 0 & \cdots & \cdots & \cdots & \cdots & \cdots & 0 \\
0 & -1 & 1 & 0 & \cdots & \cdots & \cdots & \cdots & 0 \\
\vdots & \vdots & \vdots & \vdots & \vdots & \vdots & \vdots & \vdots & \vdots \\
0 & \cdots & \cdots & \cdots & \cdots & \cdots & 0 & -1 & 1
\end{array}\right]_{[(M-1) N+M(N-1) \times M N}
$$

$\boldsymbol{F}=\left[\begin{array}{c}f_{1,1} \\ f_{2,1} \\ \vdots \\ f_{M, N}\end{array}\right]_{M N \times 1}$

$$
\boldsymbol{G}=\left[\begin{array}{c}
G^{x} \\
G^{y}
\end{array}\right]=\frac{1}{2}\left[\begin{array}{c}
\left(s_{1,2}^{x}+s_{1,1}^{x}\right)\left(x_{1,2}-x_{1,1}\right) \\
\left(s_{2,2}^{x}+s_{2,1}^{x}\right)\left(x_{2,2}-x_{2,1}\right) \\
\vdots \\
\left(s_{M, N}^{x}+s_{M, N-1}^{x}\right)\left(x_{M, N}-x_{M, N-1}\right) \\
\left(s_{2,1}^{y}+s_{1,1}^{y}\right)\left(y_{2,1}-y_{1,1}\right) \\
\left(s_{3,1}^{y}+s_{2,1}^{y}\right)\left(y_{3,1}-y_{2,1}\right) \\
\vdots \\
\left(s_{M, N}^{y}+s_{M-1, N}^{y}\right)\left(y_{M, N}-y_{M-1, N}\right)
\end{array}\right]_{[(M-1) N+M(N-1)] \times 1}
$$


Eq. (4) can be solved to get $\boldsymbol{F}$ as,

$$
\boldsymbol{F}=\left(\boldsymbol{D}^{T} \boldsymbol{D}\right)^{-1} \boldsymbol{D}^{T} \boldsymbol{G}
$$

where $\boldsymbol{D}^{T}$ denotes the transpose of $\boldsymbol{D}$.

The TFLI method is widely used for its simplicity and reliability. However, in Eq. (3) the lefthand side is approximately equal to the right-hand side. Using Taylor's theorem, it can be shown that this method has an error of $O\left(h^{3}\right)$ where $h$ is the interval between two neighboring grid points [24]. In recent years, further research in this area has been reported specifically to improve the accuracy of the method. Huang et al. [23] proposed Iterative Finite-difference-based Leastsquares Integration (IFLI) method. They applied iterative compensations to enhance TFLI method. However, IFLI method is much more computationally time consuming although the accuracy of reconstruction is higher. Li et al. [24] proposed a more accurate and less time consuming approach, the Higher-order Finite-difference-based Least-squares Integration (HFLI) method. It applies Taylor's theorem and considers two additional adjacent slopes during iteration resulting in an algorithmic error of $O\left(h^{5}\right)$. In this method, the $\boldsymbol{G}$ matrix is expressed as ${ }^{1}$ :

$\boldsymbol{G}=\left[\begin{array}{l}G^{x} \\ G^{y}\end{array}\right]$

\footnotetext{
${ }^{1}$ A correction to the expression reported in Ref. [26] has been incorporated in these expressions.
} 
$G^{x}=\frac{1}{24}\left[\begin{array}{c}12\left(s_{1,2}^{x}+s_{1,1}^{x}\right)\left(x_{1,2}-x_{1,1}\right) \\ 12\left(s_{2,2}^{x}+s_{2,1}^{x}\right)\left(x_{2,2}-x_{2,1}\right) \\ \vdots \\ 12\left(s_{M, 2}^{x}+s_{M, 1}^{x}\right)\left(x_{M, 2}-x_{M, 1}\right) \\ \left(-s_{1,4}^{x}+13 s_{1,3}^{x}+13 s_{1,2}^{x}-s_{1,1}^{x}\right)\left(x_{1,3}-x_{1,2}\right) \\ \left(-s_{2,4}^{x}+13 s_{2,3}^{x}+13 s_{2,2}^{x}-s_{2,1}^{x}\right)\left(x_{2,3}-x_{2,2}\right) \\ \vdots \\ \left(-s_{M, 4}^{x}+13 s_{M, 3}^{x}+13 s_{M, 2}^{x}-s_{M, 1}^{x}\right)\left(x_{M, 3}-x_{M, 2}\right) \\ \left(-s_{1,5}^{x}+13 s_{1,4}^{x}+13 s_{1,3}^{x}-s_{1,2}^{x}\right)\left(x_{1,4}-x_{1,3}\right) \\ \vdots \\ \left(-s_{M, 5}^{x}+13 s_{M, 4}^{x}+13 s_{M, 3}^{x}-s_{M, 2}^{x}\right)\left(x_{M, 4}-x_{M, 3}\right) \\ \vdots \\ \left.+13 s_{M, N-1}^{x}+13 s_{M, N-2}^{x}-s_{M, N-3}^{x}\right)\left(x_{M, N-1}-x_{M, N-2}\right) \\ 12\left(s_{1, N}^{x}+s_{1, N-1}^{x}\right)\left(x_{1, N}-x_{1, N-1}\right) \\ 12\left(s_{2, N}^{x}+s_{2, N-1}^{x}\right)\left(x_{2, N}-x_{2, N-1}\right) \\ \vdots \\ 12\left(s_{M, N}^{x}+s_{M, N-1}^{x}\right)\left(x_{M, N}-x_{M, N-1}\right) \\ \left(-s_{M, N}^{x}\right)\end{array}\right]_{[(M-1) N] \times 1}$

$G^{y}=\frac{1}{24}\left[\begin{array}{c}12\left(s_{2,1}^{y}+s_{1,1}^{y}\right)\left(y_{2,1}-y_{1,1}\right) \\ \left(-s_{4,1}^{y}+13 s_{3,1}^{y}+13 s_{2,1}^{y}-s_{1,1}^{y}\right)\left(y_{3,1}-y_{2,1}\right) \\ \left(-s_{5,1}^{y}+13 s_{4,1}^{y}+13 s_{3,1}^{y}-s_{2,1}^{y}\right)\left(y_{4,1}-y_{3,1}\right) \\ \vdots \\ \left(-s_{M-1,1}^{y}+13 s_{M-2,1}^{y}+13 s_{M-3,1}^{y}-s_{M-2,1}^{y}\right)\left(y_{M-2,1}-y_{M-3,1}\right) \\ 12\left(s_{M, 1}^{y}+s_{M-1,1}^{y}\right)\left(y_{M, 1}-y_{M-1,1}\right) \\ 12\left(s_{2,2}^{y}+s_{1,2}^{y}\right)\left(y_{2,2}-y_{1,2}\right) \\ \left(-s_{4,2}^{y}+13 s_{3,2}^{y}+13 s_{2,2}^{y}-s_{1,2}^{y}\right)\left(y_{3,2}-y_{2,2}\right) \\ \vdots \\ \left(-13 s_{M-2,2}^{y}+13 s_{M-3,2}^{y}-s_{M-2,2}^{y}\right)\left(y_{M-2,2}-y_{M-3,2}\right) \\ 12\left(s_{M, 2}^{y}+s_{M-1,2}^{y}\right)\left(y_{M, 2}^{y}-y_{M-1,2}\right) \\ 12\left(s_{2,3}^{y}+s_{1,3}^{y}\right)\left(y_{2,3}-y_{1,3}\right) \\ \left(-s_{4,3}^{y}+13 s_{3,3}^{y}+13 s_{2,3}^{y}-s_{1,3}^{y}\right)\left(y_{3,3}-y_{2,3}\right) \\ \vdots \\ 12\left(s_{M, N}^{y}+s_{M-1, N}^{y}\right)\left(y_{M, N}-y_{M-1, N}\right)\end{array}\right]_{[M(N-1)] \times 1}$ 


\section{Algorithm Verification}

To verify the accuracy of HFLI method independently, a function $f(x, y)$ was defined as follows:

$$
f(x, y)=(x+y) \cos \left(x^{2}-y^{2}\right)+2 \sin \left(x^{2}+y\right) \cos (x y+y)
$$

The chosen range for $x$ and $y$ coordinates for this demonstration were from -1.5 to $1.5 \mathrm{~mm}$ with an interval of $0.01 \mathrm{~mm}$, resulting in a $300 \times 300$ data grid. As evident from Fig. 5(a) the function includes significant complexities in its features. The corresponding slope contours in the $x$ - and $y$-directions are shown in Fig. 5(d) and (e), respectively. After removing the piston errors [28], the reconstructed function using HFLI algorithm is shown in Fig. 5(b). The integration errors shown in Fig. 5(c) indicate the high accuracy of HFLI method. The integration errors on the edges are somewhat higher because of the lack of two adjacent slopes on the edges as evident from Eq. (10) and (11). The mean values of errors in the region away from the edges are essentially zero or negligible.

\section{Wafer subjected to central loading}

The feasibility of HFLI method used in conjunction with r-DGS to obtain mechanically induced out-of-plane deformation maps for a deformed thin silicon wafer subjected to central loading was considered next. A single-face polished $360 \mu \mathrm{m}$ thick silicon wafer of dimeter $50.8 \mathrm{~mm}$ was used in the experiment. The unpolished face was bonded to a thick steel washer with a circular aperture of $30 \mathrm{~mm}$ using a slow curing epoxy in order to simulate a thin circular plate with a circular clamped boundary. Another steel plate fitted with a micrometer at its center was positioned directly behind the silicon wafer. These two steel plates were secured in a cylindrical lens holder. A target plate, decorated with random black and white speckles, was placed horizontally at $90^{\circ}$ to the silicon wafer. The target was illuminated by two cool LED lamps to avoid air currents. A beam splitter was placed at $45^{\circ}$ to both the silicon wafer and the target plate. It was also at $45^{\circ}$ to the optical axis of the camera (see Fig. 6). The distance $(\Delta)$ between the silicon wafer surface and the target plate along the optical axis was $62 \mathrm{~mm}$.

A Nikon D100 digital SLR camera was focused on the target via the polished face of the silicon wafer. The camera was fitted with a 70-300 mm macro lens and an adjustable bellows. A small aperture $\left(F^{\#}=22\right)$ was selected for recording the speckles with a good focus. The distance 
between the silicon wafer and the end of lens $(L)$ was $1403 \mathrm{~mm}$. When the silicon wafer was under no load, an 8-bit reference image was recorded with a resolution of $1504 \times 1000$ pixels (1 pixel $=43.9 \mu \mathrm{m})$. Then, known central out-of-plane displacements, $w$, were imposed on the silicon wafer using the micrometer tip and speckle images corresponding to the deformed states of the wafer were recorded. By correlating each of these speckle images with the reference image, the local displacements $\delta_{y: x}$ in the region of interest were measured. During correlation (using ARAMIS image analysis software), the images were sub-divided into $20 \times 20$ pixels with 10 pixel overlap in the $x$ - and $y$-directions, resulting in $97 \times 133$ matrix of data points in the field.

Two sets of orthogonal surface slope contours, $\frac{\partial w}{\partial x}$ and $\frac{\partial w}{\partial y}$ measured from r-DGS are shown in Figs. 7 for two imposed central deflections, $4 \pm 1 \mu \mathrm{m}$ and $10 \pm 1 \mu \mathrm{m}$. For completeness, contours of $\frac{\partial w}{\partial r}\left(=\sqrt{\left(\frac{\partial w}{\partial x}\right)^{2}+\left(\frac{\partial w}{\partial y}\right)^{2}}\right)$ are also shown, where $r$ is the radial distance from the center of the wafer. The contours are plotted with increments of 50 and 100 micro-radians in the two cases, respectively. It can be observed that the magnitude of contours of $\frac{\partial w}{\partial x}$ and $\frac{\partial w}{\partial y}$ are symmetric about $x=0$ and $y=0$ with a high concentration of contours near the loading point. The contours of $\frac{\partial w}{\partial r}$ show circular symmetry, again with a higher concentration of contours around the loading point. As expected, slopes are zero along the circular edge (and beyond) of the Si wafer in the glued portion and near the loading point with a gradual transition in the intermediate region.

The measurements from r-DGS are compared directly with the closed-form solutions for an elastic thin circular plate with a clamped boundary subjected to a centrally applied deflection using,

$$
\frac{\partial w}{\partial y}=\frac{4 y w}{R^{2}} \log \left(\frac{\sqrt{x^{2}+y^{2}}}{R}\right)
$$

where $w$ is the out-of-plane deformation and $R$ is the radius of the circular plate. 
The comparisons shown in Fig. 8 are made between experimental and analytical data sets along the $y$-axis for different magnitudes of out-of-plane deformations. The least count of the micrometer used being $2 \mu \mathrm{m}$, results from $w=2 \mu \mathrm{m}$ is also added to this comparison. It can be readily observed that there is a good agreement between the two data sets with percentage error decreasing as imposed deflection is increased.

The reconstructed 3D surface computed using integration of surface slope data and HFLI computations is plotted in Fig. 9(a) for the $10 \mu \mathrm{m}$ deflection case. Fig. 9(b) shows the corresponding contours of out-of-plane displacements $(w)$ at $0.5 \mu \mathrm{m}$ increments. The circular contours in Fig. 9(b) demonstrates that the shape of the reconstructed figure matches well with the reality of the experiment both qualitatively and quantitatively. (Note that for all the plots in Figs. 7 and 9, the origin is made to coincide with the loading point.) The peak value of the height of the reconstructed 3D surface is $10.41 \mu \mathrm{m}$ with an acceptable error of $4.1 \%$. (The reconstruction for the smaller imposed deflection of $4 \mu \mathrm{m}$, not shown here for brevity, led to a peak value of $4.81 \mu \mathrm{m}$, a deviation of $20.2 \%$. Given the uncertainty of the imposed deflection of $\pm 1 \mu \mathrm{m}$, the reconstructed peak value is deemed reasonable despite a higher error.) The errors are attributed to a combined effect of (a) micrometer backlash and human errors while imposing the out-of-plane displacement, (b) the non-uniformity and finite compliance of the epoxy adhesive used to 'clamp' the Si wafer to the steel plate, (c) errors due to DGS measurements as well as integration errors in the HFLI method. Although it is beneficial to isolate each of these effects, it is difficult to do so practically. It should also be mentioned that the reconstructed surface along the glued boundary of the wafer shows non-zero deflection (approximately $1 \mu \mathrm{m}$ instead of zero). This is attributed to a combination of reconstruction errors as well as compliance of the epoxy glue layer alluded to earlier.

The accuracy of HFLI method relies on the interval between two neighboring grid points. And, this interval in turn depends on the choice of sub-image size and pixel overlap used during correlation of the reference and deformed images while implementing r-DGS. Further, the overlap was selected for achieving correlation at all points in the region of interest with minimum noise in the plotted data. Therefore, the trade-offs if any due to different interval choices on HFLI outcome was examined. Fig. 10 shows the measured contours of $\frac{\partial w}{\partial y}$ for $20 \times$ 
20 pixels sub-images with three different overlaps of $0,10,15$ pixels and the corresponding reconstructed surfaces. Higher number of overlapping pixels resulted in the interval $(h)$ between two neighboring grid points getting smaller. In Fig. 10, it can be observed that the slope contours are generally in good agreement with each other with minor differences between the slope contours near the wafer boundary where the slopes are expected to be zero. However, the interval $(h)$ for 0 pixel overlap and 15 pixel overlap cases are different by a factor of 2 and 0.5 relative to the 10 pixel overlap case. The reconstructed surfaces for each of these cases are shown in Fig. 10. (Note that the plots appear darker as the grid density increases.) The difference of the peak values of out-of-plane displacement for the three cases relative to the imposed value range between $3.2 \%$ and $4.5 \%$ suggesting the robustness of the reconstruction. The height of the reconstructed surface for the zero pixel overlap case is the closest to $10 \mu \mathrm{m}$, however, it is less accurate due to the larger interval between neighboring grid points. As a result, 10 pixel overlap was judged appropriate for subsequent analyses.

\section{Wafer subjected to eccentric loading}

The measurement of slopes and reconstruction of out-of-plane deformations of a silicon wafer subjected to eccentric loading was attempted next. In this experiment, again out-of-plane displacements, $w=4 \pm 1 \mu \mathrm{m}$ and $10 \pm 1 \mu \mathrm{m}$, were imposed on the silicon wafer eccentrically at a distance of $5 \mathrm{~mm}$ away from the center of the wafer along the $y$-axis. Otherwise, the optical setup in this experiment was the same one used in the previous experiment. A $20 \times 20$ sub-image size with 10 pixel overlap was also used here to correlate the images in the deformed and undeformed states to get displacements $\delta_{y: x}$.

The two orthogonal surface slope contours, $\frac{\partial w}{\partial x}$ and $\frac{\partial w}{\partial y}$ for each of these imposed displacements are shown in Figs. 11. The contours of $\frac{\partial w}{\partial r}$ are also provided. As in the central loading case, all the contours are plotted with increments of 50 and 100 micro-radians for the 4 $\mu \mathrm{m}$ and $10 \mu \mathrm{m}$ cases, respectively. It can be observed that the contours of $\frac{\partial w}{\partial x}$ and $\frac{\partial w}{\partial y}$ are symmetric about $x=0$, but asymmetric about $y=0$. Accordingly, the contours of $\frac{\partial w}{\partial x}, \frac{\partial w}{\partial y}$ and 
$\frac{\partial w}{\partial r}$ are relatively crowded in the positive $y$-direction. The noise at the circular clamped boundary and its vicinity in the figures are attributed to measurement errors in r-DGS. The reconstructed 3D surface calculated from HFLI is plotted in Figs. 12(a). Fig. 12(b) shows the corresponding contours of out-of-plane displacement $(w)$ at $0.5 \mu \mathrm{m}$ increments. These plots demonstrate that the shape of the reconstructed surface match the reality in the experiment. Again, in Figs. 11 and 12, $(0,0)$ is made to coincide with the loading point. The peak value of the height of the reconstructed 3D surface is approximately $10.53 \mu \mathrm{m}$. Based on the elasticity theory for a circular plate loaded by an eccentric concentrated force [31], the peak value of deflection is $10.16 \mu \mathrm{m}$. Further, the location of the theoretical peak value is at $(x=0, y=-0.8)$ or $0.8 \mathrm{~mm}$ away from the loading point along the $-y$ direction. Compared with this theoretical value, the reconstruction error is an acceptable 3.6\%. The sources of errors are the same as the ones discussed in the previous experiment. Evidently, the reconstructed surface shows asymmetry as expected along with an obvious tilt due to higher stresses and deformation suffered by the epoxy adhesive layer along the boundary close to the loading point relative to the far away edges. That is, the reconstructed shape is tilted towards the positive $y$-direction, in agreement with the load point eccentricity relative to the center of the wafer.

As in the symmetric loading case, surface reconstruction for $w=4 \mu \mathrm{m}$ case was also carried out and the results are not shown in favor of brevity. The peak value of the height of the reconstructed 3D surface in this case was approximately $5.12 \mu \mathrm{m}$ with an error of $26 \%$ relative to the theoretical value $4.06 \mu \mathrm{m}$. Again, due to the uncertainty of the imposed deflection of $\pm 1 \mu \mathrm{m}$, the reconstructed peak value was deemed reasonable.

\section{Dynamic mode-I crack propagation in transparent PMMA}

The previous two experiments have demonstrated the feasibility of coupling r-DGS methodology with HFLI approach for reconstructing surface profile due to mechanical deformation. Next, the work is extended to transmission-mode DGS (or, t-DGS) to extract stress field data from measured orthogonal stress gradient data to study dynamic crack propagation in brittle polymers such as PMMA. First, the dynamic crack growth in PMMA sheets was studied using t-DGS technique in conjunction with ultrahigh-speed digital photography. The schematic of the experimental setup employed is shown in Fig. 13. A Hopkinson pressure bar (a 25 mm dia., 
$1.8 \mathrm{~m}$ long 7075-T6 aluminum bar) was used for subjecting a pre-notched ( $40^{\circ} \mathrm{V}$-notch) PMMA specimen to wedge-loading using a gas-gun and a striker bar (a $25 \mathrm{~mm}$ dia. and $225 \mathrm{~mm}$ long aluminum rod). A Cordin-550 ultrahigh-speed digital camera with 32 independent $1000 \times 1000$ pixels sensors was used for recording the speckle images during the dynamic fracture event. The specimen was at a distance of approximately $850 \mathrm{~mm}$ in front of the camera. A target plate decorated with random black and white speckles was placed behind the specimen at a distance $(\Delta)$ $29.3 \mathrm{~mm}$ away from the mid-plane of the specimen. The region of interest in this study was in the crack tip neighborhood (52 mm x $52 \mathrm{~mm}$ ). A pair of heavy dots (Fig. 14) marked on the target plate were used to relate the dimension on the image to the actual specimen/target dimensions. Prior to loading the specimen, a set of 32 images of the speckles were recorded at 200,000 frames per second through the specimen in its undeformed state. Next, without altering any of the camera settings, the specimen was dynamically loaded by impacting the long-bar with the striker bar (striker velocity $\sim 14 \mathrm{~m} / \mathrm{sec}$ ). The impact event triggered recording of a second set of 32 images in the deformed state at the same framing rate. Thus, a total of 32 pairs of images in the deformed and undeformed (reference) states were recorded at $5 \mu$ s intervals between successive images. Two representative speckle images in the region of interest, one in the undeformed state and the other in the deformed state are shown in Fig. 14(a) and (b), respectively. The speckle pattern shown in Fig. 14(b) corresponds to a time instant $95 \mu$ sec after crack initiation at the initial notch tip. It can be seen that the speckles are noticeably distorted in the near vicinity of the propagating crack tip (in the deformed image) whereas they seem largely unaffected in the far-field. The corresponding image pairs from each sensor were correlated separately. Further details of the experiment are previously reported by Sundaram and Tippur [29]. During image correlation ${ }^{2}$, each image was segmented into $25 \times 25$ pixels sub-images. An overlap of 20 pixels (i.e., a step size of 5 pixels) was used during analysis. This resulted in $194 \times$ 194 matrix of data points in the region of interest for each of the two orthogonal displacement fields. The corresponding angular deflections of light rays were subsequently determined using the known distance $\Delta$ between the specimen and the target planes. Fig. 15 shows the angular deflection plots at three select time instants. The crack velocity history for such a crack growth has been previously reported in [29]. Using the angular deflection fields along the $x$-direction

\footnotetext{
${ }^{2}$ The image correlation was carried out in r-DGS also using the image analysis software ARAMIS ${ }^{\circledR}$.
} 
mode-I and mode-II SIFs were evaluated using an over-deterministic least-squares analysis based on the asymptotic expressions for the measured stress gradients (See [29] for details),

$\phi_{x}(t)=C_{\sigma} B\left[\begin{array}{c}-\frac{1}{2} r_{l}^{-\frac{3}{2}}\left\{\begin{array}{l}f\left(V ; C_{L} ; C_{S}\right) A_{1}(t) \cos \left(\frac{3 \theta_{l}}{2}\right) \\ +g\left(V ; C_{L} ; C_{S}\right) D_{1}(t) \sin \left(-\frac{3 \theta_{l}}{2}\right)\end{array}\right\} \\ +\sum_{N=2}^{\infty}\left\{\begin{array}{l}A_{N}(t)\left(\frac{N}{2}-1\right) r_{l}^{\left(\frac{N}{2}-2\right)} \cos \left(\left(\frac{N}{2}-2\right) \theta_{l}\right) \\ +D_{N}(t)\left(\frac{N}{2}-1\right) r_{l}^{\left(\frac{N}{2}-2\right)} \sin \left(\left(\frac{N}{2}-2\right) \theta_{l}\right)\end{array}\right\}\end{array}\right]$

where $f$ and $g$ denote functions of instantaneous crack velocity, and $\left(r_{l}, \theta_{l}\right)$ denote the contracted crack tip polar coordinates for a growing crack, $C_{\sigma}$ is the elasto-optic constant of the material $\left(1.08 \times 10^{-10} \mathrm{~m}^{2} / \mathrm{N}\right)$, and $B$ is its initial thickness. Further, $\left(r_{l}, \theta_{l}\right)$ can be expressed in the local Cartesian coordinates $\left(x^{\prime}, y^{\prime}\right)$ as, $r_{l}=\left\{\left(x^{\prime}\right)^{2}+\alpha_{L}^{2}\left(y^{\prime}\right)^{2}\right\}^{1 / 2}$ and $\theta_{l}=\tan ^{-1}\left(\frac{\alpha_{L} y^{\prime}}{x^{\prime}}\right)$. The coefficients of $A_{1}(t)$ and $D_{1}(t)$ in the asymptotic series are related to the mode-I and mode-II Stress Intensity Factors $(\mathrm{SIF}) K_{I}(t)$ and $K_{I I}(t)$, respectively, as $A_{1}(t)=K_{I}(t) \sqrt{2 / \pi}$ and $D_{1}(t)=K_{I I}(t) \sqrt{2 / \pi}$. The functions $f$ and $g$ are [29],

$$
f\left(V ; C_{L}, C_{S}\right)=\left(\frac{1+v}{1-v}\right) \frac{\left(1+\alpha_{S}^{2}\right)\left(1-\alpha_{L}^{2}\right)}{4 \alpha_{L} \alpha_{S}-\left(1+\alpha_{S}^{2}\right)^{2}} \text { and } g\left(V ; C_{L}, C_{S}\right)=\left(\frac{1+v}{1-v}\right) \frac{2 \alpha_{S}\left(1-\alpha_{L}^{2}\right)}{4 \alpha_{L} \alpha_{S}-\left(1+\alpha_{S}^{2}\right)^{2}}
$$

where $\alpha_{L}=\left[1-\frac{\rho(1-v)}{2 \mu} V^{2}\right]^{\frac{1}{2}}$ and $\alpha_{S}=\left[1-\frac{\rho}{\mu} V^{2}\right]^{\frac{1}{2}}$ for plane stress. Data in the region $(0.25<$ $r / B<0.75)$ and $\left(-135^{\circ}<\theta<135^{\circ}\right)$ near the crack tip was used for analysis. Fig. 16 shows the plot of SIF history evaluated. As this is a mode-I crack growth, $K_{I I}$ is expected to be relatively small if not zero. Further, it can be seen that the $K_{I}$ remains approximately same throughout the window of observation. 
Using the SIFs evaluated from the stress gradient fields, the $\left(\sigma_{x x}+\sigma_{y y}\right)$ fields around the moving crack tip were obtained using the mode-I K-dominant expression for stresses as [30],

$$
\left(\sigma_{x x}+\sigma_{y y}\right)=\frac{K_{I}(t)}{\sqrt{2 \pi r}} f(\theta=0, V)
$$

where $V$ is the crack velocity,

$$
f(\theta ; V)=\frac{1}{D} \frac{2 \alpha_{L}^{2}\left(1+\alpha_{S}^{2}\right)}{\sqrt{\gamma_{L}}} \cos \left(\frac{\theta_{L}}{2}\right), D=4 \alpha_{S} \alpha_{L}-\left(1+\alpha_{S}^{2}\right)^{2}, \alpha_{S ; L}=\sqrt{1-\left(\frac{V}{C_{S ; L}}\right)^{2}},
$$

$\gamma_{S ; L}=\sqrt{1-\left(\frac{V \sin \theta}{C_{S ; L}}\right)^{2}}, \tan \theta_{S ; L}=\alpha_{S ; L} \tan \theta$ and $C_{S ; L}$ denote the shear and longitudinal wave speeds of the material. Contour plots of constant $\left(\sigma_{x x}+\sigma_{y y}\right)$ for a time instant $100 \mu$ sec after crack initiation are shown in Fig. 17(a).

The $\left(\sigma_{x x}+\sigma_{y y}\right)$ fields around the moving crack tip were also obtained by integrating the measured orthogonal stress gradient fields and HFLI method. Fig. 17(b) shows the $\left(\sigma_{x x}+\sigma_{y y}\right)$ fields from HFLI algorithm at a select time instant of $100 \mu \mathrm{sec}$ during dynamic crack growth corresponding to the stress gradient contours shown in Fig. 17(a). It can be seen that both the stress fields have qualitative similarities near the crack tip vicinity. However, it should be emphasized that the reconstructed stresses, unlike the theoretical counterpart, encompass all the far-field effects (or, the higher order terms). In light of this, the contours do deviate both qualitatively and quantitatively from the ones shown in Fig. 17(a) based on K-dominant (oneterm description) in Eq. (16). The 3D surface plot of reconstructed stress around the crack tip is shown in Fig. 18 that indicates severe stress amplification in the immediate crack tip vicinity.

To compare the $\left(\sigma_{x x}+\sigma_{y y}\right)$ fields obtained from these two methods more effectively, $\left(\sigma_{x x}+\sigma_{y y}\right)$ values were evaluated at select directions namely $\left(r, \theta=0^{\circ}\right)$ and $\left(r, \theta=45^{\circ}\right)$ relative to the crack tip ( $\theta=0^{\circ}$ denotes crack growth direction) are plotted in Fig. 19. We can see a good 
agreement between the two plots in the region $(0.25<r / B<0.75)$ where the data was selected for performing the least-squares analysis. For $r / B>0.75$ the $K$-dominance is lost and for $r / B<$ 0.25 there is significant stress triaxiality whereas contours based on Eq. (16) are based on 2D (plane stress) assumptions.

\section{Conclusions}

In this work, the feasibility of surface profile evaluation and stress field mapping from reflection and transmission mode Digital Gradient Sensing (DGS) methods are demonstrated. This is accomplished by coupling DGS output with a Higher-order Finite-difference-based Leastsquares Integration (HFLI) scheme implemented in the Southwell configuration. DGS being a digital image correlation-based method capable of providing two orthogonal gradients simultaneously, it naturally lends itself to the implementation of algorithms in the Southwell configuration.

First, the surface profile measurements are demonstrated successfully using a 'clamped' reflective Si wafer subjected to out-of-plane concentrated load applied centrally and eccentrically in two separate sets of experiments. The imposed deflection has been recovered using the HFLI scheme with good accuracy relative to the accuracy of the micrometer used. For $10 \pm 1 \mu \mathrm{m}$ imposed deflection cases, the recovered values show approximately $5 \%$ deviation from the expected value. The reconstruction has also revealed non-zero out-of-plane deflections in the 'clamped' regions of the plate due to the compliance of the epoxy adhesive layer. The phenomenon is pronounced in the eccentric loading case with the glued edge closer to the loading point show higher deflections relative to the far-away edge. The analysis of data with different data intervals show that the method is quite robust and the peak value variations are relatively negligible among the three cases considered.

Further, reconstruction of $\left(\sigma_{x x}+\sigma_{y y}\right)$ stress fields from stress gradients is demonstrated for the case of a dynamically growing crack in a transparent polymer (PMMA) sheet. For a time instant of $100 \mu \mathrm{sec}$ after crack initiation, the stress gradient fields used in conjunction with HFLI algorithm resulted in obtaining stress fields with qualitative similarities to the theoretical (or, the so-called K-dominant) counterparts. The comparison was limited because the crack tip stress intensity factors were evaluated by analyzing measured gradients close to the crack tip in 
conjunction with the asymptotic series expansion based on 2D assumptions. Accordingly, only the first coefficient $\left(K_{\mathrm{I}}\right)$ of the expansion was used to recreate the theoretical stress fields. The reconstructed/experimental stresses and the theoretical values agree quite well in the crack tip vicinity although they deviate from each other due to the higher order effects far away from the crack tip and due to triaxial effects very close to the crack tip.

\section{Acknowledgments}

The corresponding author acknowledges partial support of this research through Department of Defense grants W31P4Q-14-C-0049 and ARMY-W911NF-16-1-0093.

\section{References}

[1] D. Malacara-Doblado and I. Ghozeil, "Hartmann, Hartmann-Shack, and Other Screen Tests," in Optical Shop Testing, Wiley, 2007.

[2] M. Strojnik, G. Paez and M. Mantravadi, "Lateral shear interferometers," in Optical Shop Testing, Wiley, 2007.

[3] R. Ragazzoni, "Pupil plane wavefront sensing with an oscillating prism," J. Mod. Opt., vol. 43, pp. 289-293, 1996.

[4] S. Ettl, J. Kaminski, M. Knauer and G. Häusler, "Shape reconstruction from gradient data," Appl Opt, vol. 47, pp. 2091-2097, 2008.

[5] A. Assa, A. A. Betser and J. Politch, "Recording slope and curvature contours of flexed plates using gratings shearing interferometer," Appl Opt, vol. 16, no. 9, pp. 2504-2513, 1997.

[6] T. Y. Kao and F. P. Chiang, "Family of grating techniques of slope and curvature measurements for static and dynamic flexure of plates," Opt Eng, vol. 21, no. 4, pp. 721742, 1982.

[7] H. V. Tippur, "Simultaneous and real-time measurement of slpoe and curvature fringes in thin structures using shearing interferometry," Opt Eng, vol. 43, pp. 3014-3020, 2004.

[8] H. V. Tippur, S. Krishnaswamy and A. J. Rosakis, "Optical mapping of crack tip deformation using the methods of transmission and reflection coherent gradient sensing-a study of crack tip K-Dominance," Int J Fract, vol. 52, pp. 91-117, 1991.

[9] R. Ritter, "Reflection moire methods for plate bending studies," Opt Eng, vol. 21, pp. 663671, 1982. 
[10] M. A. Sutton and J. J. Orteu, Image correlation for shape, motion and deformation measurements, New York, NY, USA: Springer, 2009.

[11] M. S. Kirugulige, H. V. Tippur and T. S. Denney, "Measurement of transient deformations using digital image correlation method and high-speed photography: application to dynamic fracture," Appl Opt, vol. 46, no. 22, pp. 5083-5096, 2007.

[12] T. C. Chu, W. F. Ranson, M. A. Sutton and W. H. Peters, "Application of digital image correlation techniques to experimental mechanics," Exp Mech , vol. 25, no. 3, pp. 232-244, 1985.

[13] D. J. Chen, F. P. Chiang, Y. S. Tan, H. Don and S., "Digital speckle-displacement measurement using a complex spectrum method," Appl Opt , vol. 32, no. 11, pp. 1839-1849, 1993.

[14] C. Periasamy and H. V. Tippur, "Full-field digital gradient sensing method for evaluating stress gradients in transparent solids.," Appl Opt, vol. 51, no. 12, pp. 2088-2097, 2012.

[15] C. Periasamy and H. V. Tippur, "Measurement of orthogonal stress gradients due to impact load on a transparent sheet using digital gradient sensing method.," Exp Mech, vol. 53, pp. 97-111, 2013.

[16] C. Periasamy and H. V. Tippur, "A full-field reflection-mode digital gradient sensing method for measuring orthogonal slopes and curvatures of thin structures.," Meas Sci Technol , vol. 24, p. 025202, 2013.

[17] N. E. Coleman and R. Jain, "Obtaining 3-dimensional shape of textured and specular surfaces using four-source photometry," Comput Vision Graph, vol. 18, pp. 309-328, 1982.

[18] G. Healey and R. Jain, "Depth recovery from surface normals," Int Conf Pattern Recognition, vol. 2, pp. 894-896, 1984.

[19] Z. Wu and L. Li, "A line-integration based method for depth recovery from surface normals," Comput Vision Graph, vol. 43, pp. 53-66, 1988.

[20] R. T. Frankot and R. Chellappa, "A method for enforcing integrability in shape from shading algorithms," IEEE Trans. Pattern Anal. Mach. Intell, vol. 10, pp. 439-451, 1988.

[21] R. H. Hudgin, "Wave-front reconstruction for compensated imaging," J Opt Soc Am , vol. 67, pp. 375-378, 1977.

[22] W. H. Southwell, "Wave-front estimation from wave-front slope measurements," J Opt Soc Am , vol. 70, pp. 998-1006, 1980.

[23] L. Huang and A. Asundi, "Improvement of least-squares integration method with iterative compensations in fringe reflectometry," Appl Opt, vol. 51, pp. 7459-7465, 2012. 
[24] G. Li, Y. Li, K. Liu, X. Ma and H. Wang, "Improving wave front reconstruction accuracy by using integration equations with higher-order truncation errors in the Southwell geometry," J Opt Soc Am A , vol. 2013, pp. 1448-1459, 2013.

[25] R. Klette and K. Schlüns, "Height data from gradient fields," in SPIE 2908, Machine Vision Applications, Architectures, and Systems Integration V, Boston, Massachusetts, 1996.

[26] L. Huang, M. Idir, C. Zuo, K. Kaznatcheev, L. Zhou and A. Asundi, "Comparison of twodimensional integration methods for shape reconstruction from gradient data," Opt Laser Eng , vol. 64, pp. 1-11, 2015.

[27] A. S. Jain and H. V. Tippur, "Extension of reflection-mode digital gradient sensing method for visualizing and quantifying transient deformations and damage in solids," Opt Laser Eng, vol. 77, pp. 162-174, 2016.

[28] D. Malacara, Optical shop testing, John Wiley and Sons, 1992.

[29] B. M. Sundaram and H. V. Tippur, "Dynamic Crack growth normal to an interface in Bilayered materials: an experimental study using digital gradient sensing technique," Exp Mech, Vol. 56, pp 37-57, 2016.

[30] P. C. Paris and G. C. Sih, Stress Analysis of Cracks, Fracture Toughness Testing and its Applications, Philadelphia: American Society for Testing and Materials STP 381, 1964.

[31] E. Ventsel and T. Krauthammer, "Thin Plates and Shells: Theory, Analysis and Applications”, CRC Press, 2001. 


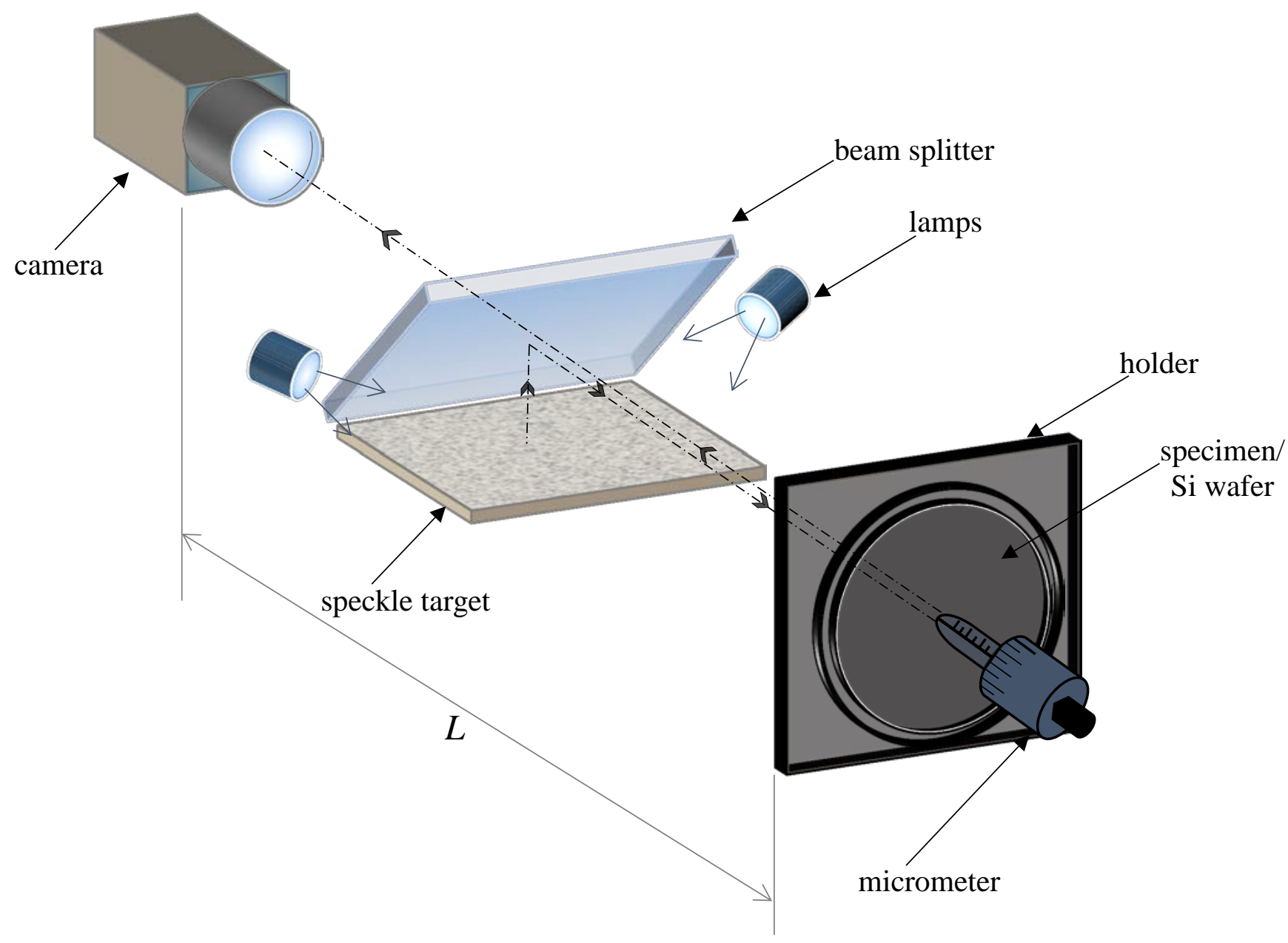

Fig. 1. Schematic of r-DGS experimental setup 


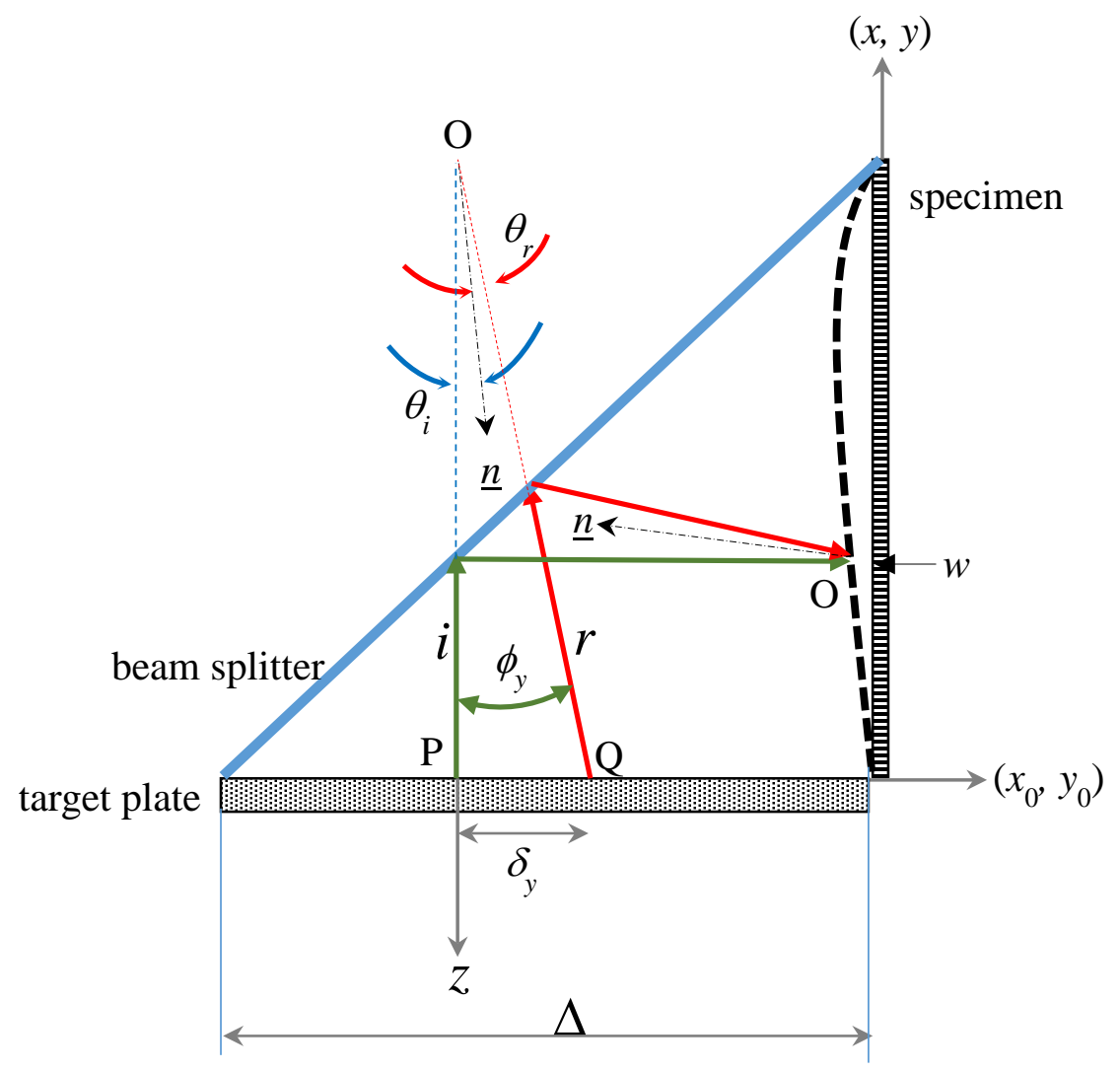

Fig. 2. Schematic explaining the working principle of r-DGS. 


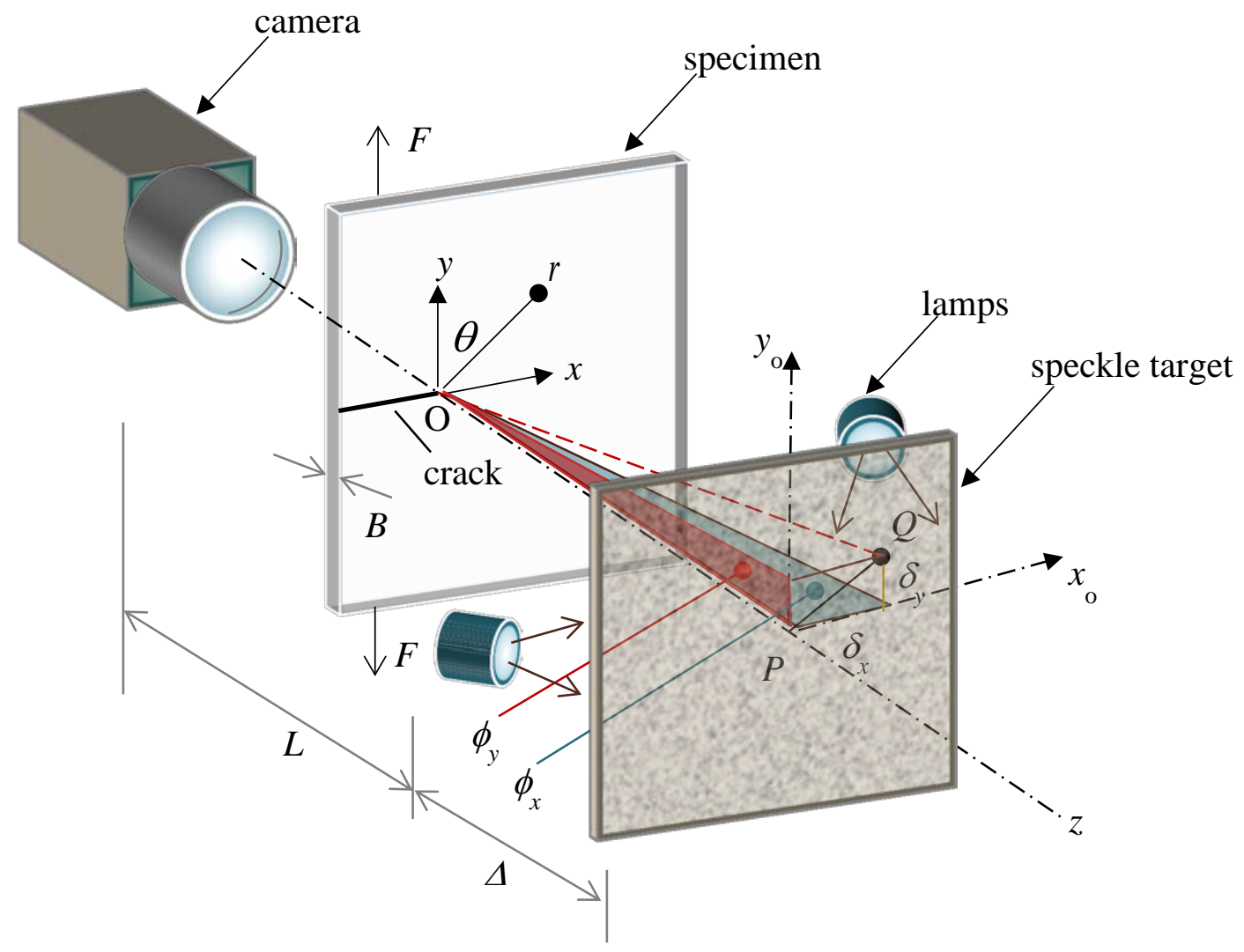

Fig. 3. The schematic of the experimental setup for t-DGS technique to determine planar stress gradients in phase objects. 


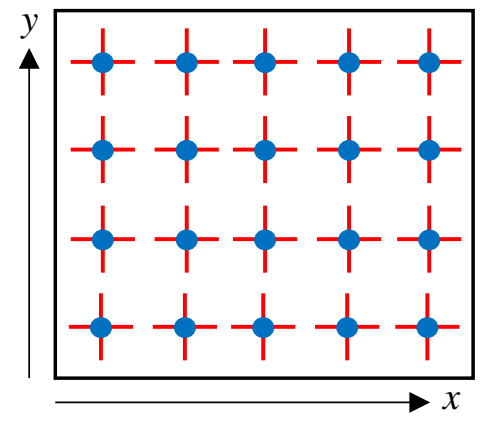

+ Position for measured surface slopes

- Position for evaluated function values

Fig. 4. Southwell grid configuration. 
(a)

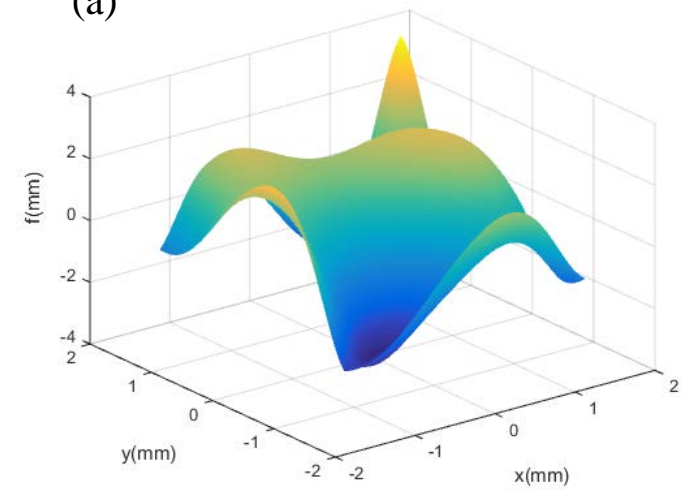

(b)
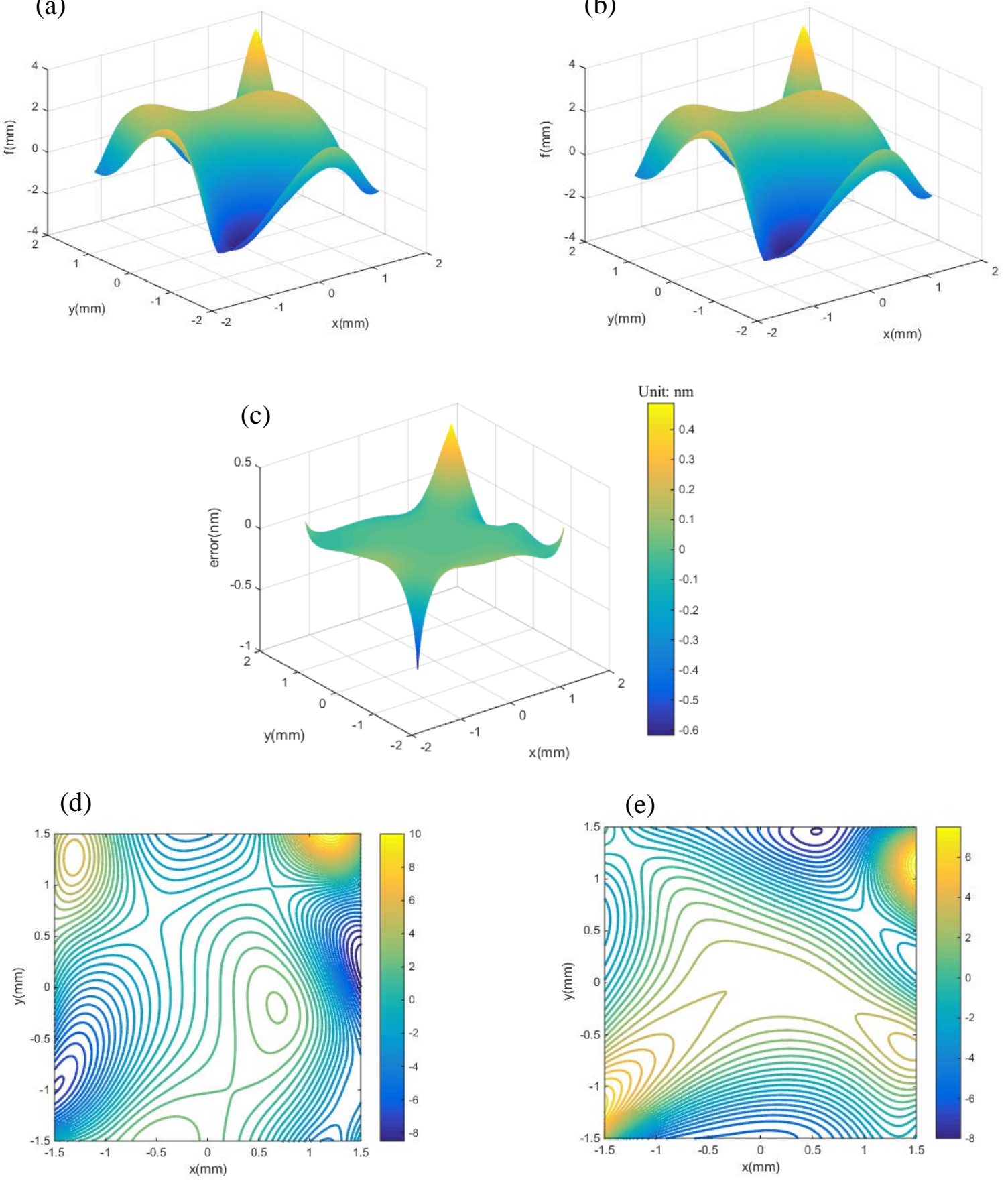

(e)

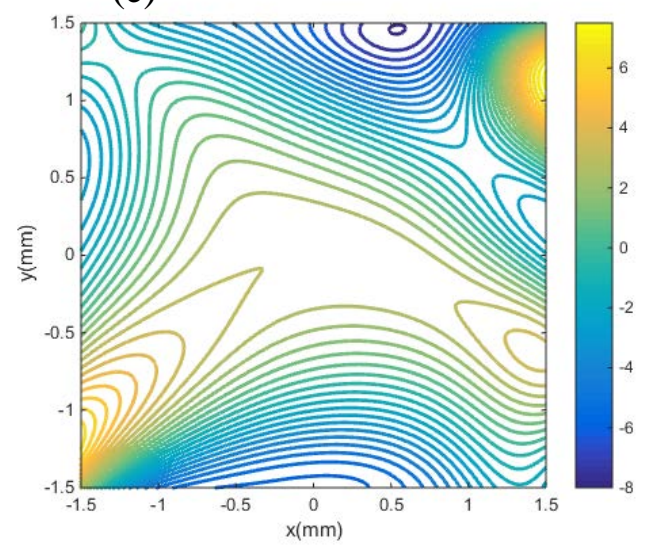

Fig. 5. Verification of HFLI method. (a) Original function, (b) reconstructed function, (c) reconstruction errors, (d) contours of derivatives of the function in the $x$-direction, (e) contours of derivatives of the function in the $y$-direction. 


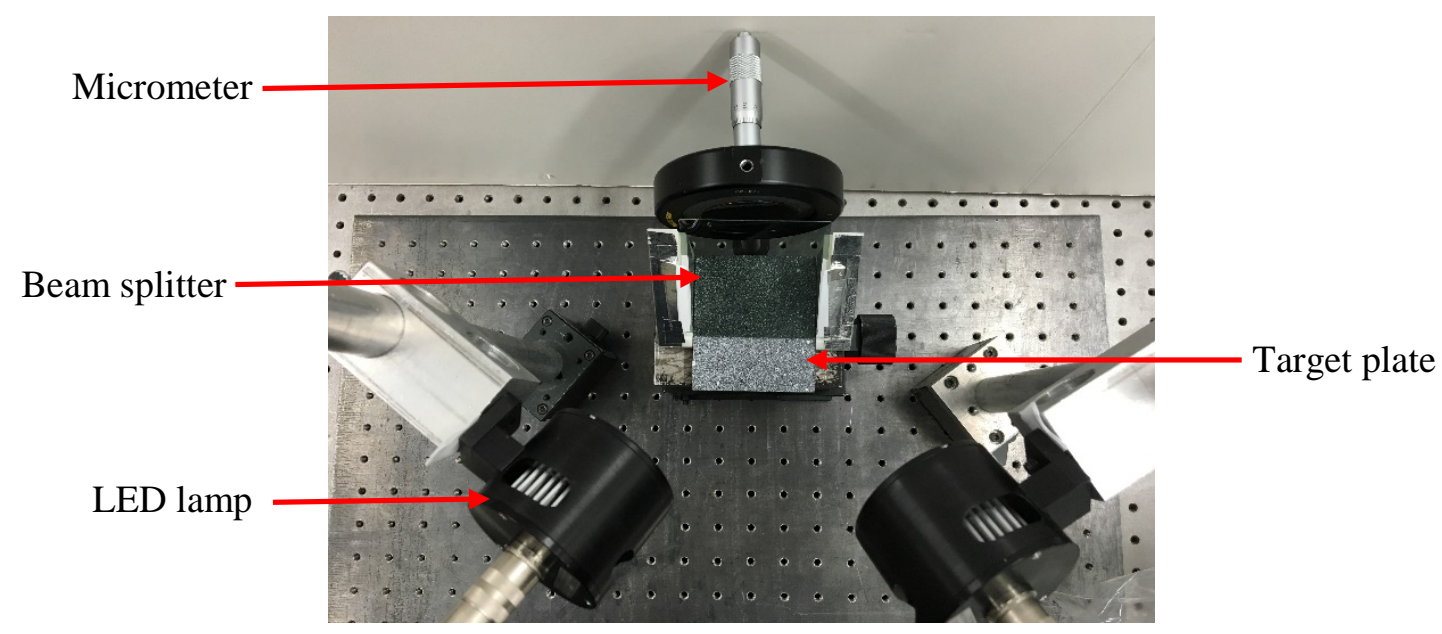

Fig. 6. The experimental setup used to study out-of-plane deformation of a silicon wafer. 

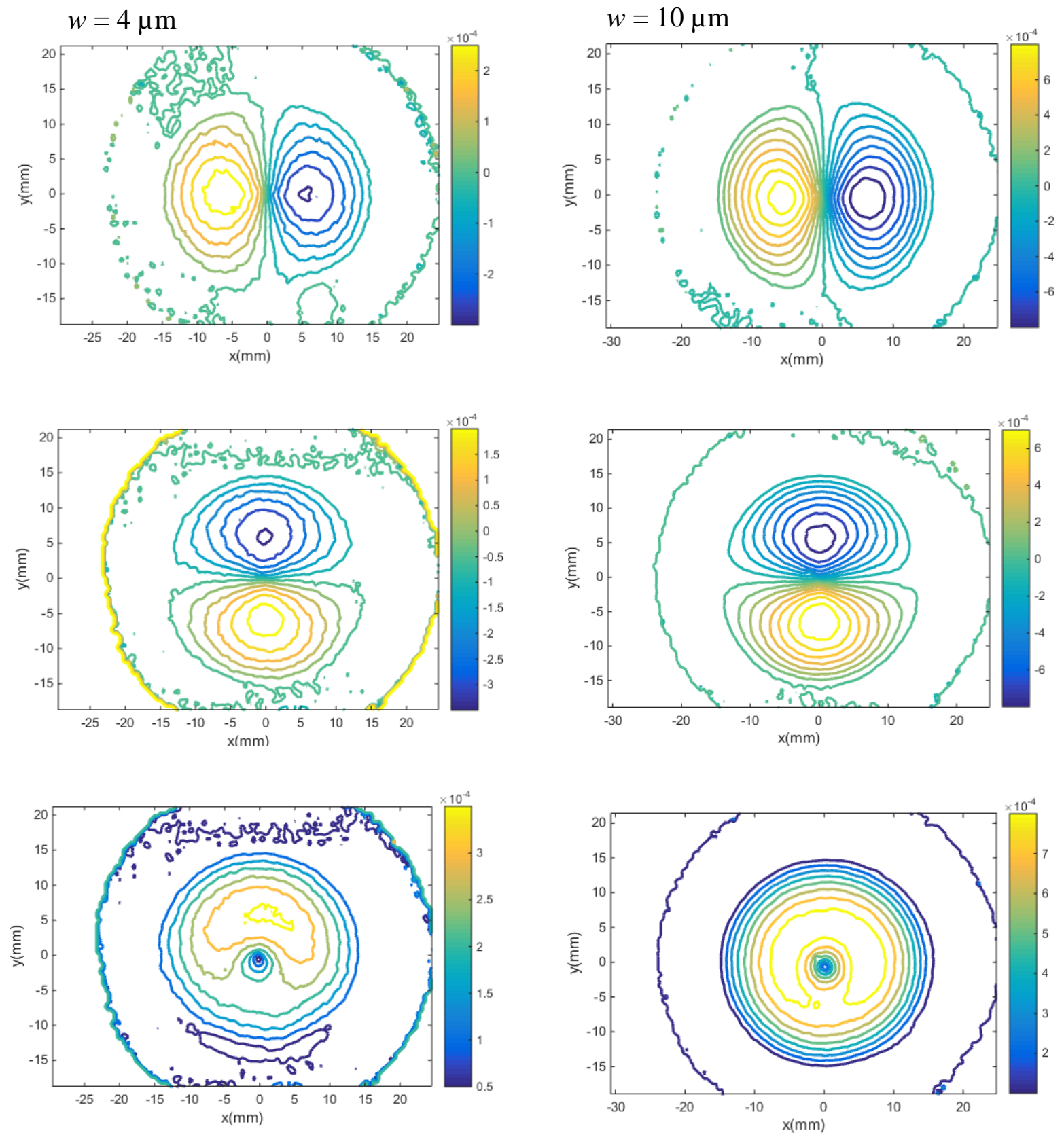

Fig. 7. Results from r-DGS for a clamped silicon wafer (30 mm dia., $360 \mu \mathrm{m}$ thick) subjected to central deflection of $4 \mu \mathrm{m}$ (left column) and $10 \mu \mathrm{m}$ (right column). Row 1: contours of $w{ }_{x}$; Row 2: contours of $w$,y Row 3: contours of $w, r$. Note: $(0,0)$ is made to coincide with the loading point. Contour increments $=0.5 \times 10^{-4} \mathrm{rad}$ and $1 \times 10^{-4} \mathrm{rad}$, respectively. 


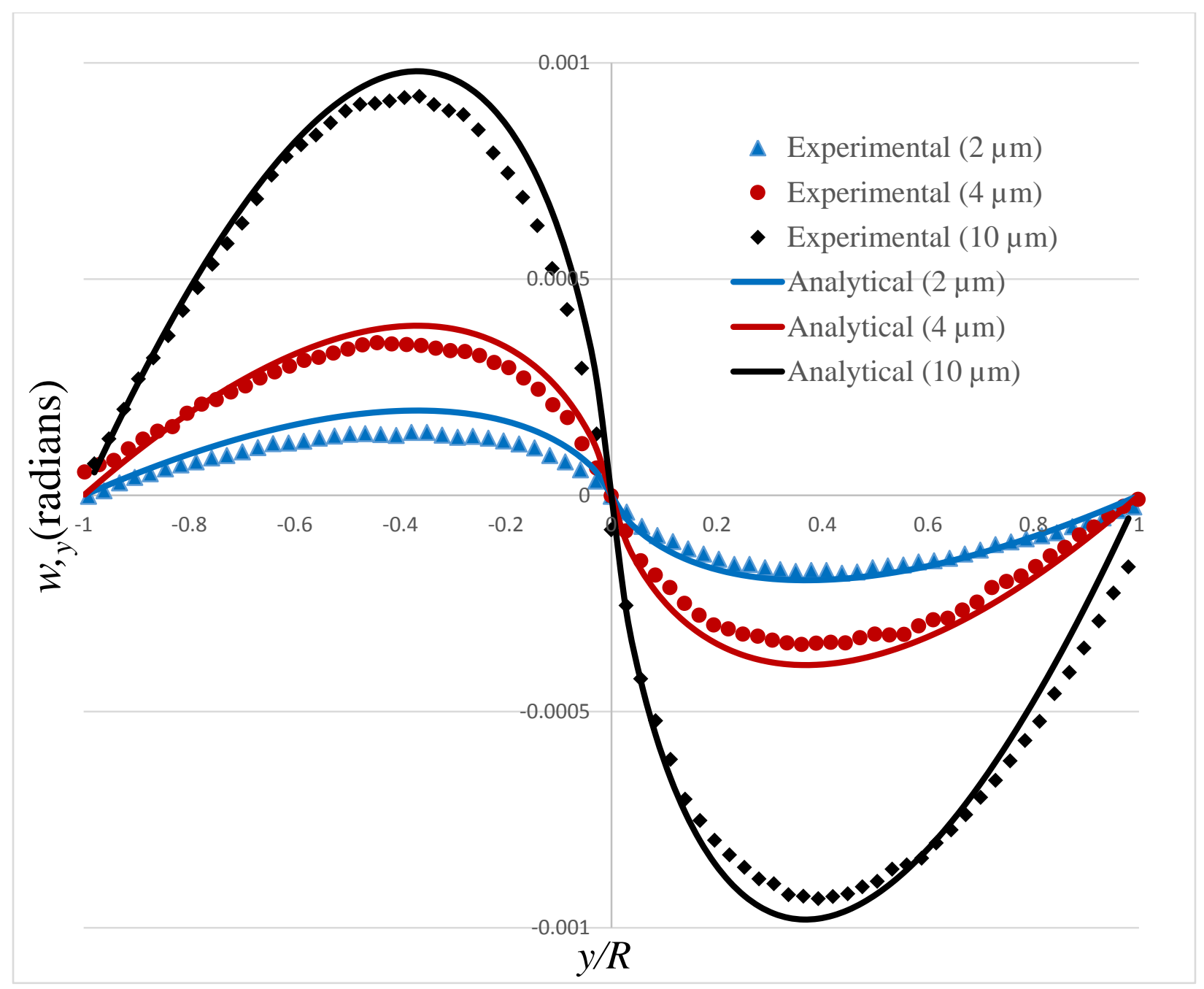

Fig. 8. Comparison of analytical data and experimental data of $\frac{\partial w}{\partial y}$ along the $y$-axis corresponding to three different imposed out-of-plane deflections. 


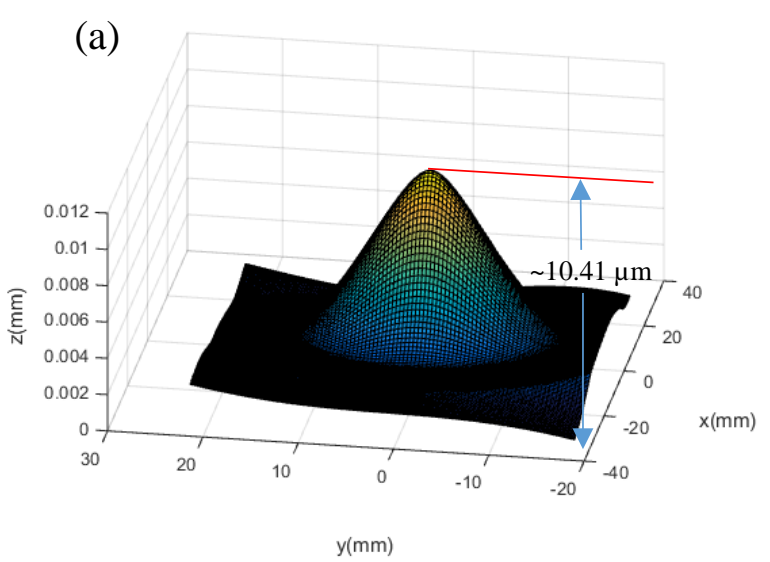

(b)

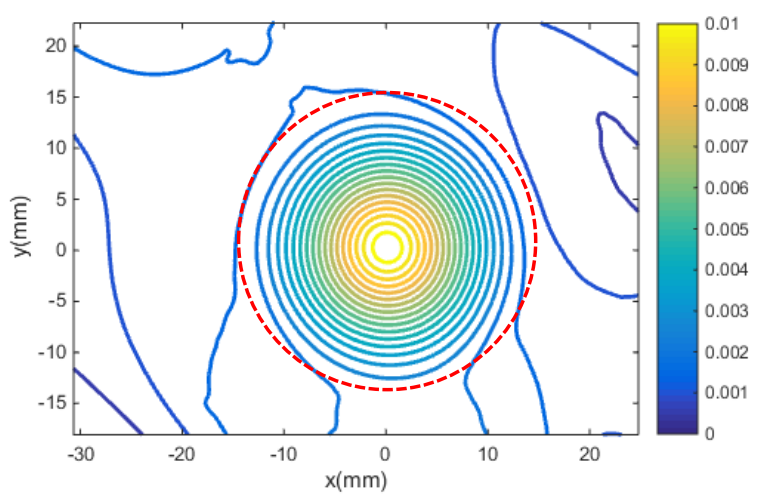

Fig. 9. Surface topography calculated via 2D integration using surface slope data and HFLI method: (a) 3D surface representation of reconstructed surface, (b) out-of-plane displacement (w) contours $(0.5 \mu \mathrm{m}$ increments). Note: $(0,0)$ is made to coincide with the loading point; red circle indicates the edge of the circular aperture of the steel washer. 

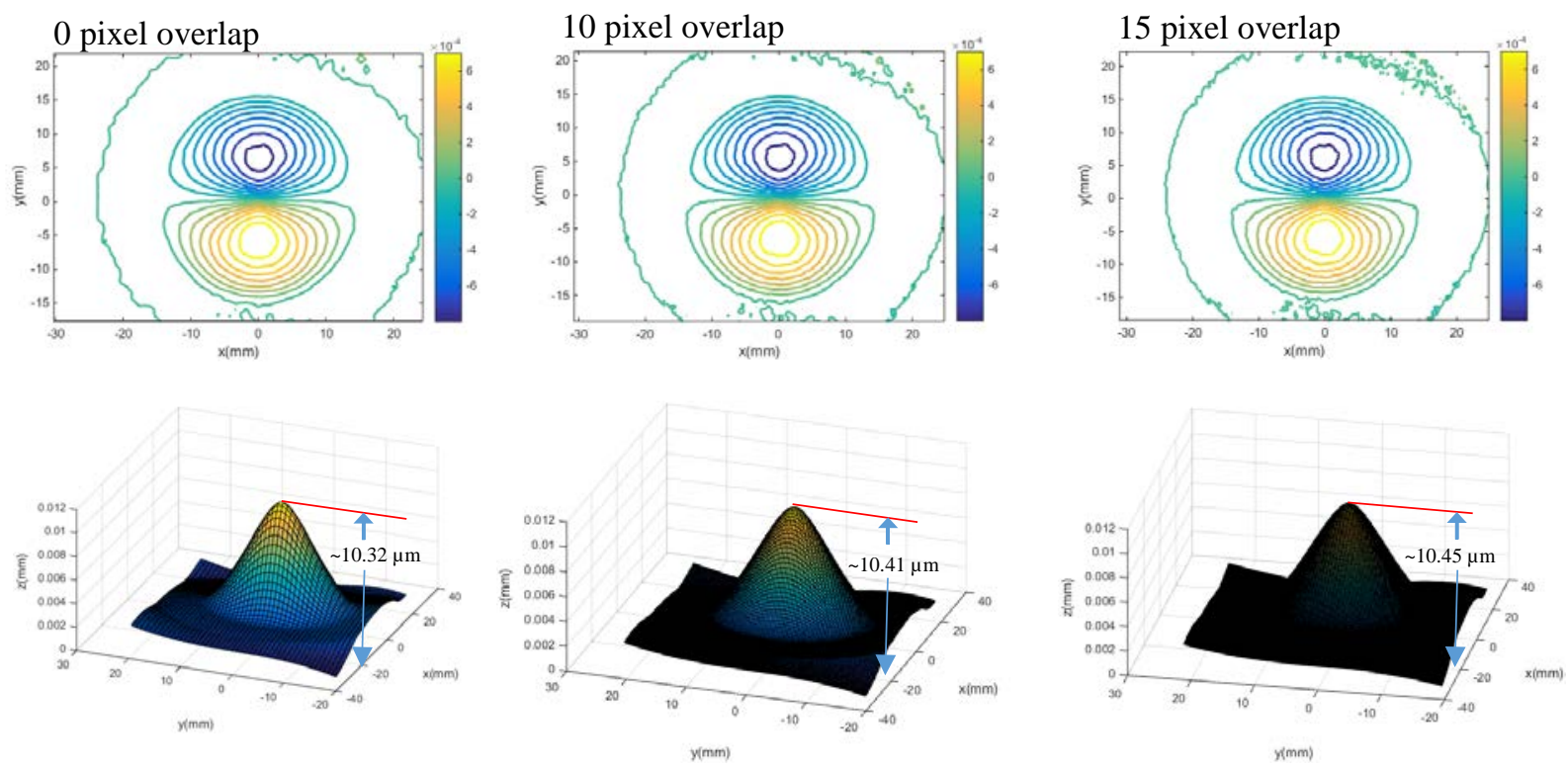

Fig. 10. Contours of $w, y$ for $20 \times 20$ pixel sub-images with three different overlap and the corresponding reconstructed figures. 

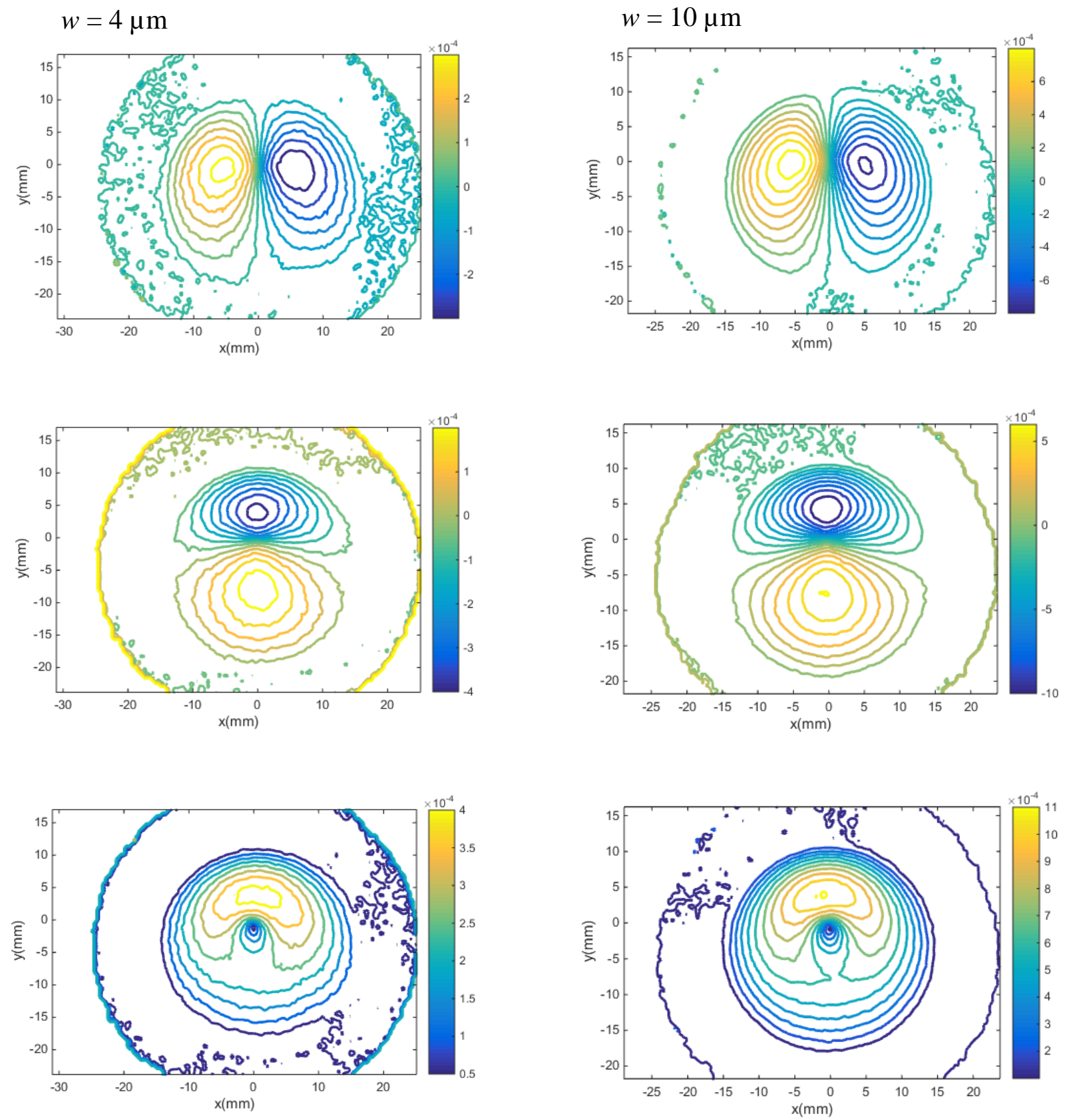

Fig. 11. Results from r-DGS for a clamped silicon wafer (30 mm dia., $360 \mu \mathrm{m}$ thick) subjected to eccentric deflection of $4 \mu \mathrm{m}$ (left column) and $10 \mu \mathrm{m}$ (right column). Row 1: contours of $w, x$; Row 2: contours of $w, y$; Row 3: contours of $w_{, r}$. Note: $(0,0)$ is made to coincide with the loading point. Contour increments $=0.5 \times 10^{-4} \mathrm{rad}$ and $1 \times 10^{-4} \mathrm{rad}$, respectively. 


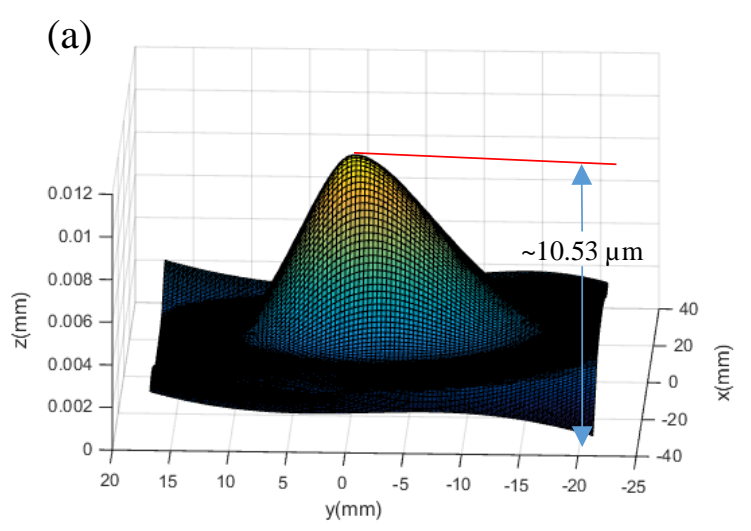

(b)

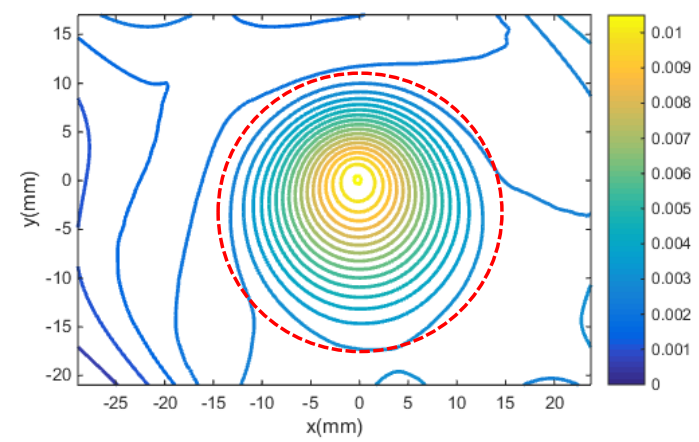

Fig. 12. Surface topography calculated via 2D integration using surface slope data and HFLI method: (a) reconstructed 3D surface, (b) out-of-plane displacement (w) contours $(0.5 \mu \mathrm{m}$ increments). Note: $(0,0)$ is made to coincide with the loading point; red circle is the circular aperture of the steel washer. 


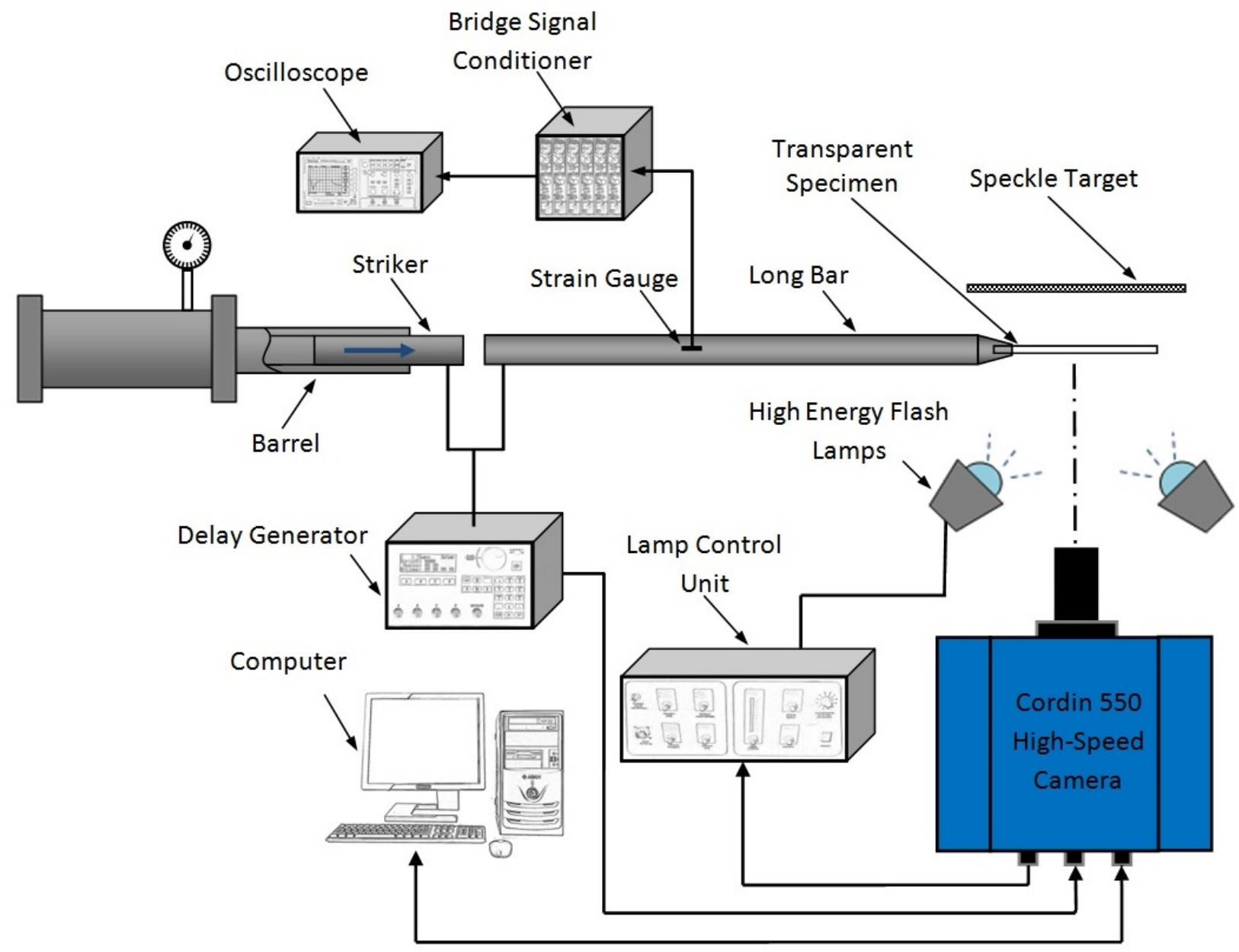

Fig. 13. Schematic of the experimental setup used for dynamic fracture study. 

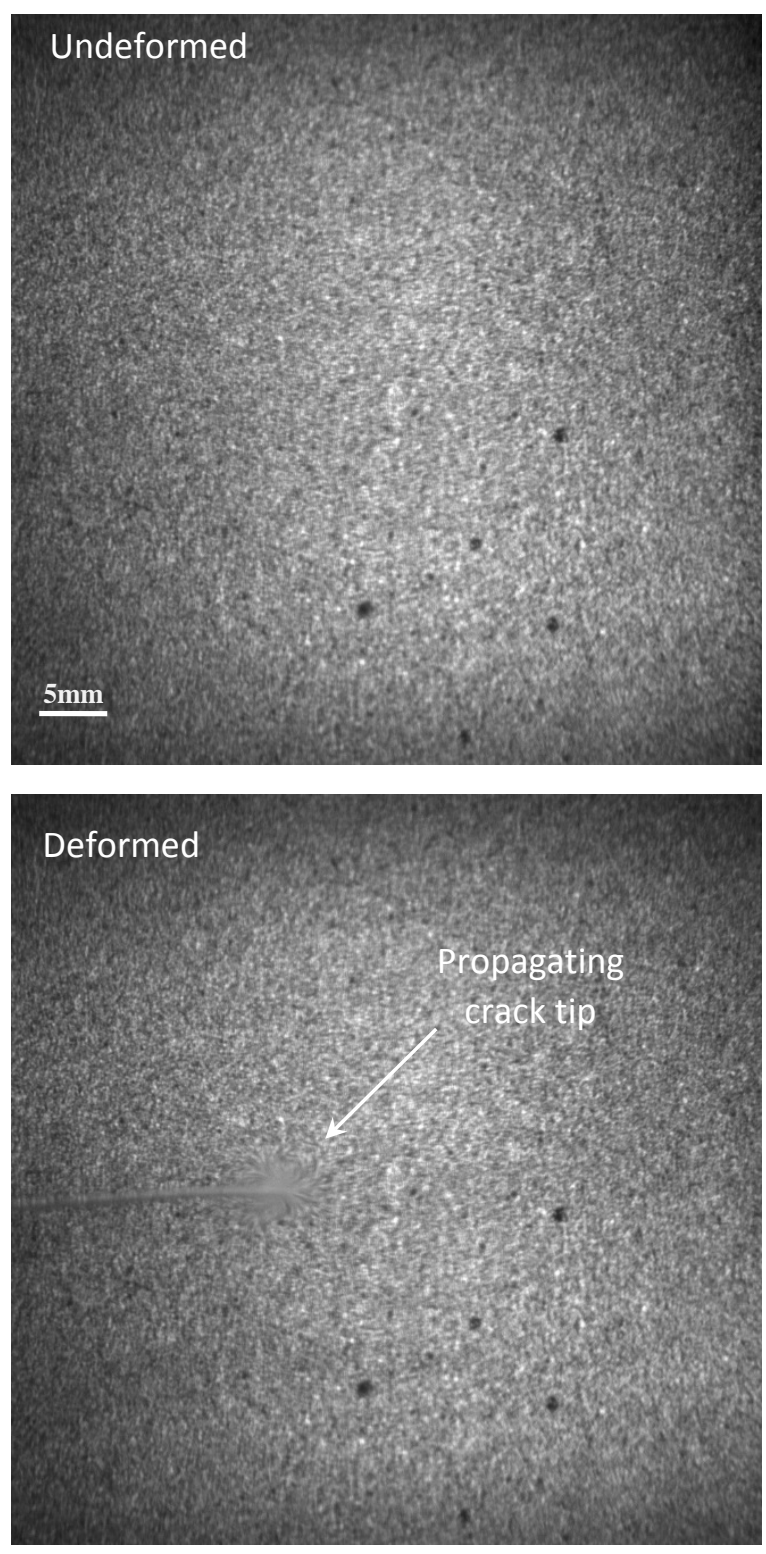

Fig. 14. Speckle images in the undeformed (top) and deformed (bottom) states recorded by the high-speed camera though the PMMA specimen. 

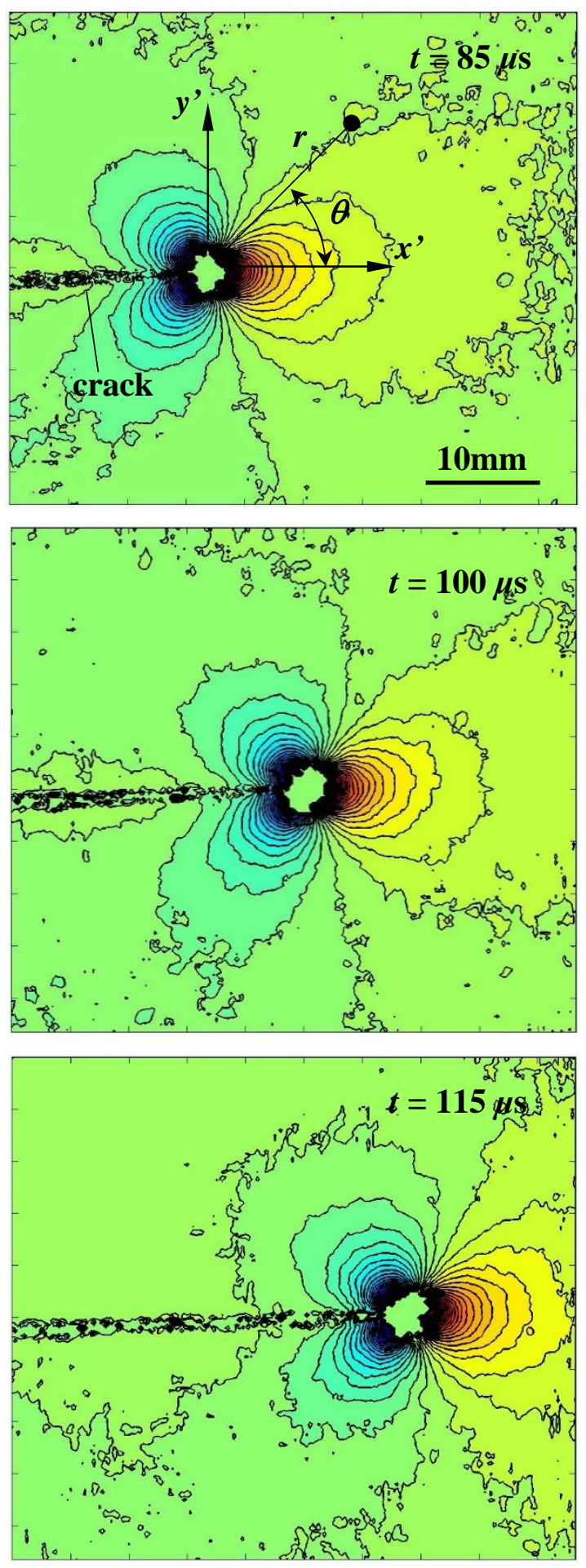

$\phi_{x}$ Contours
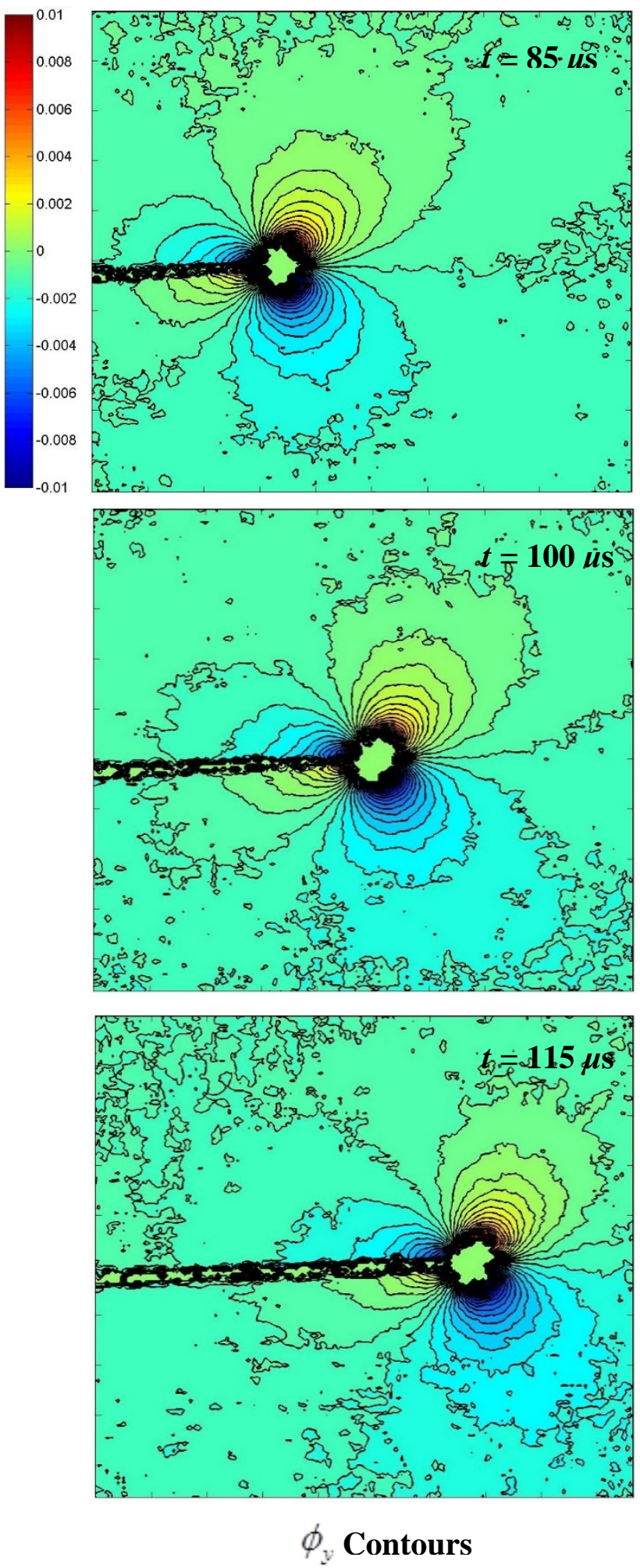
Fig. 15. Angular deflection contour plots (contour interval $=5 \times 10^{-4} \mathrm{rad}$ ) proportional to stress gradients of $\left(\sigma_{x}+\sigma_{y}\right)$ in the $x$ - and $y$-directions.

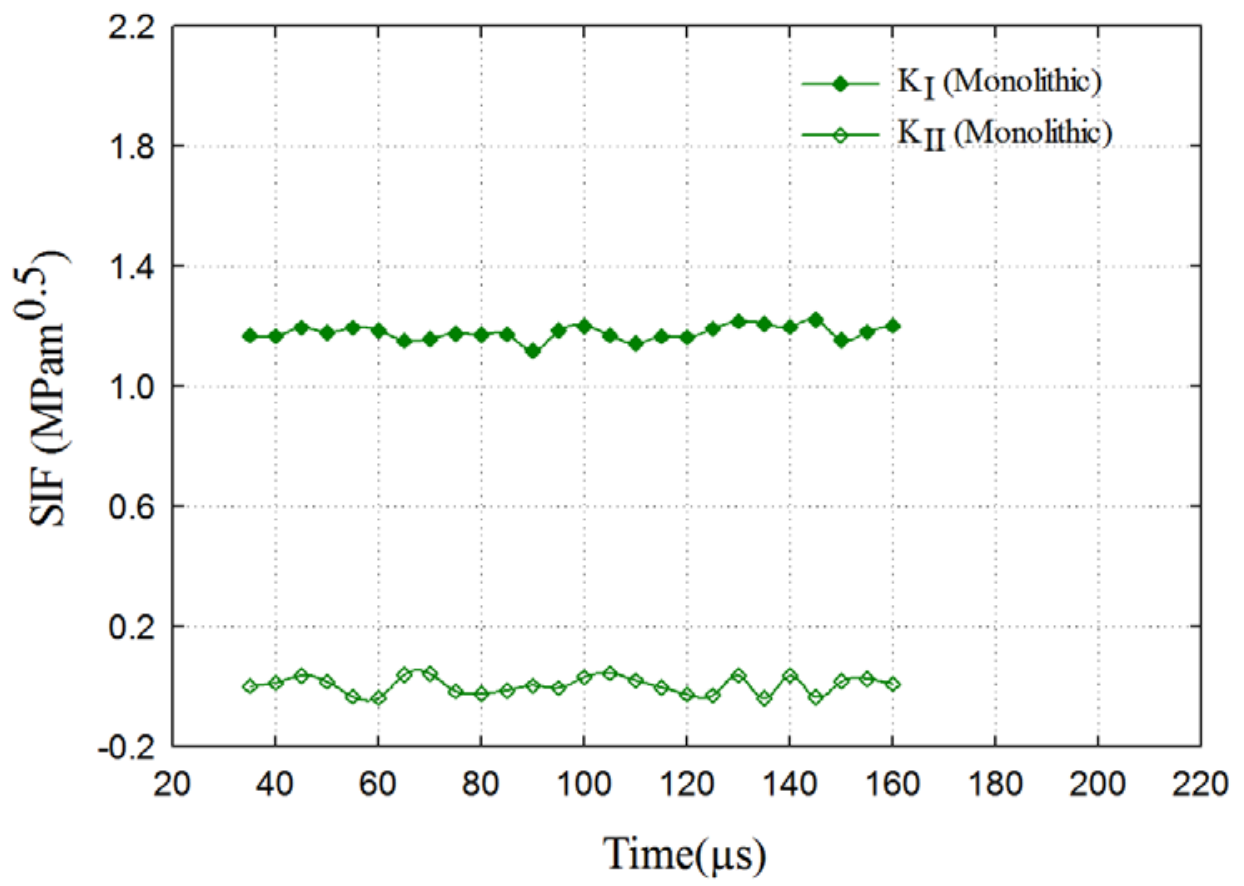

Fig. 16. Mode-I and II SIFs for a dynamically growing crack in a monolithic PMMA. 

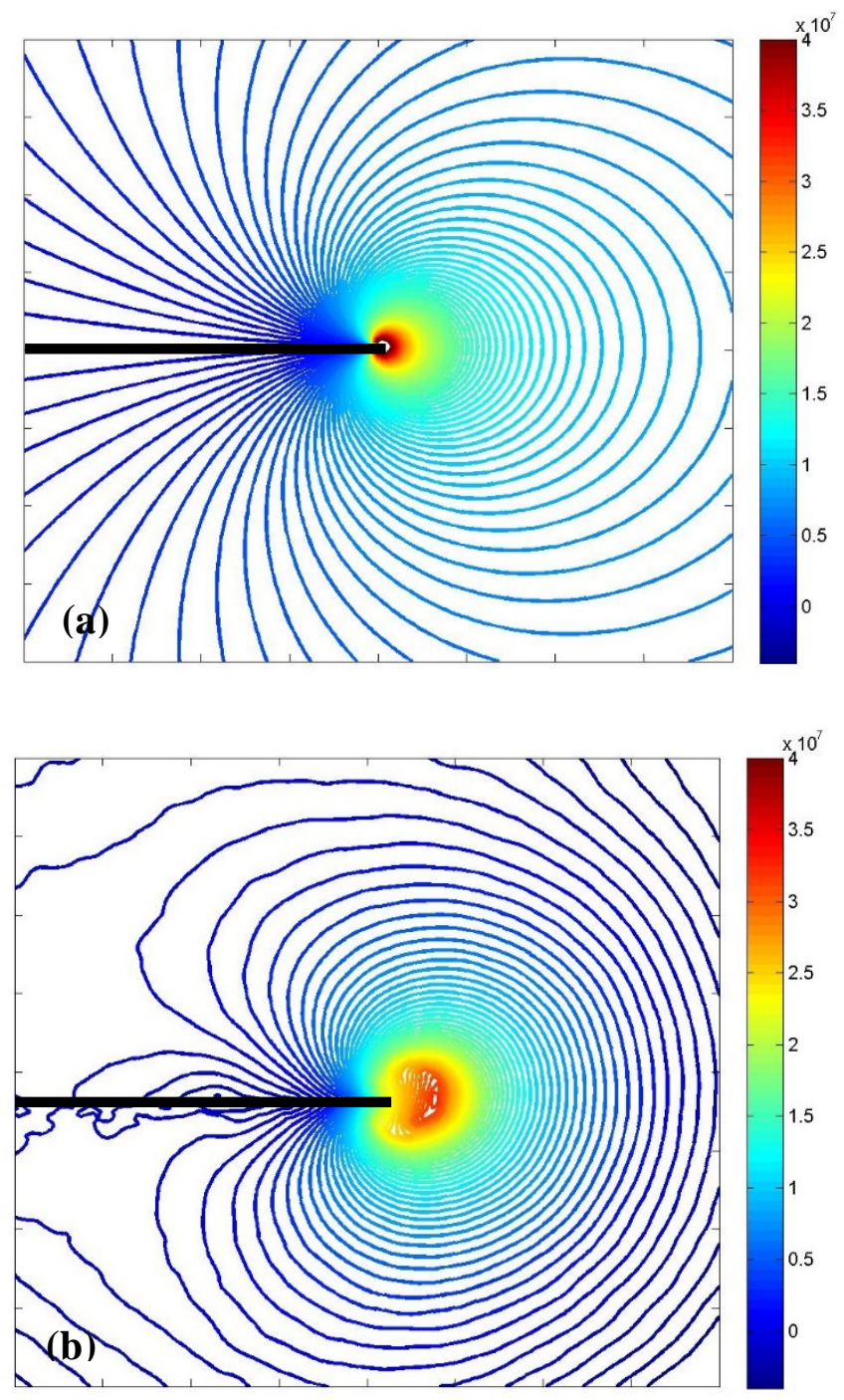

Fig. 17. $\left(\sigma_{x x}+\sigma_{y y}\right)$ fields (contour interval=0.75 MPa) obtained integration by a) using $K_{\mathrm{I}}$ and $K_{\mathrm{II}}$ measured from the DGS fields along with analytical expression for stress fields and b) using HFLI method. The black horizontal line represent the growing crack faces. 


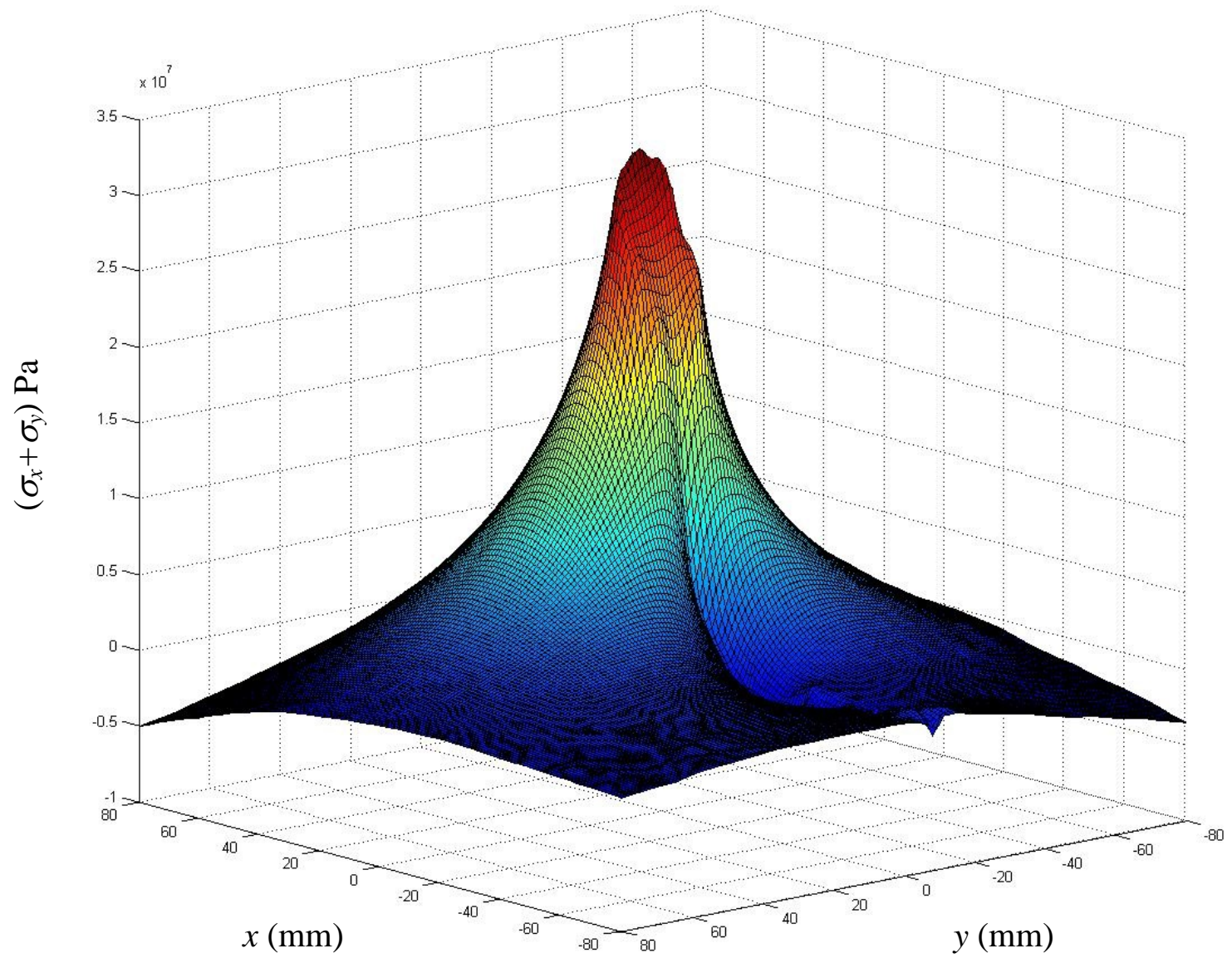

Fig. 18. The 3D surface plot of stress around a moving crack tip reconstructed using HFLI. 

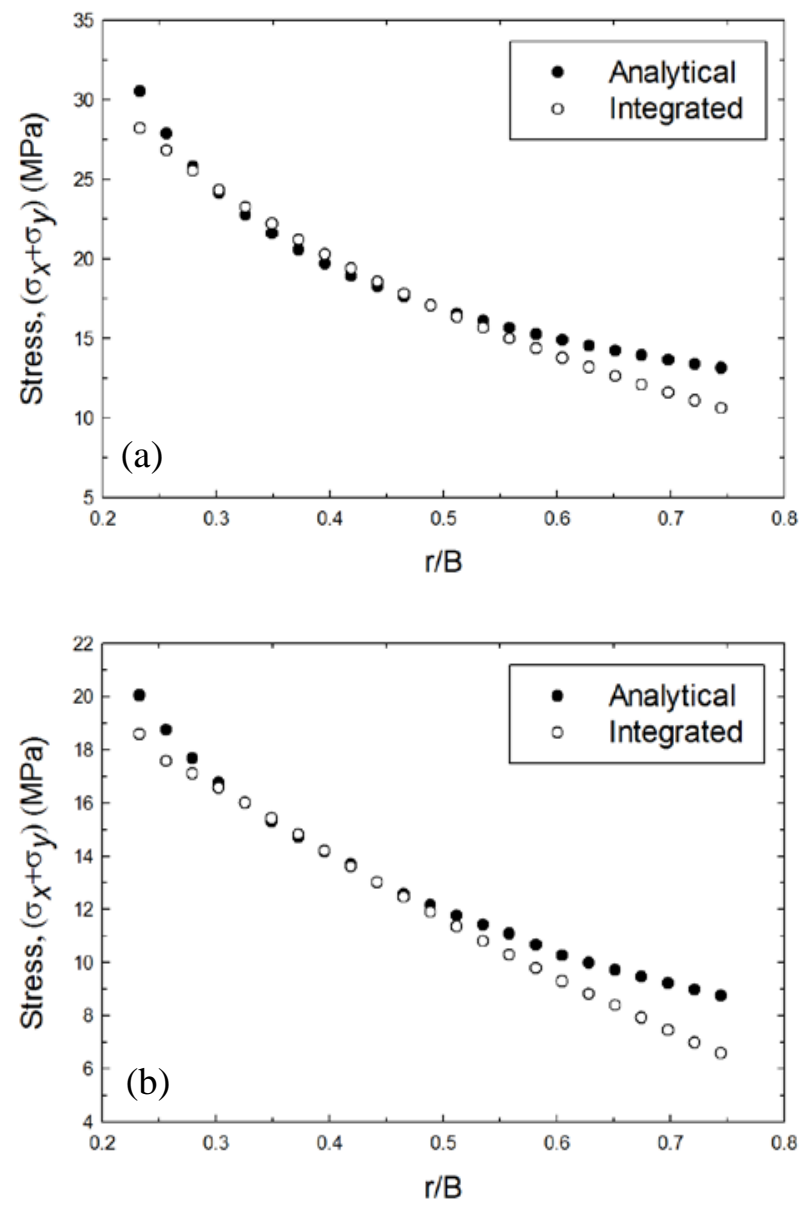

Fig. 19. The plot of stress evaluated from integration and by using K-dominant equations from the extracted SIFs for a direction of (a) $0^{\circ}$, and (b) $45^{\circ}$ from the crack growth direction. 